\title{
ANALYSIS OF ELECTROOCULOGRAM (EOG) SIGNALS IN STUDYING MYASTHENIA GRAVIS
}

\author{
by \\ Timothy Liang \\ B.Eng., Ryerson University, Toronto, Ontario, Canada, 2014 \\ A thesis \\ presented to Ryerson University \\ in partial fulfillment of the \\ requirements for the degree of \\ Master of Applied Science \\ in the program of \\ Electrical \& Computer Engineering
}

Toronto, Ontario, Canada, 2017

(C) Timothy Liang, 2017 


\section{AUTHOR'S DECLARATION FOR ELECTRONIC SUBMISSION OF A THESIS}

I hereby declare that I am the sole author of this thesis. This is a true copy of the thesis, including any required final revisions, as accepted by my examiners.

I authorize Ryerson University to lend this thesis to other institutions or individuals for the purpose of scholarly research.

I further authorize Ryerson University to reproduce this thesis by photocopying or by other means, in total or in part, at the request of other institutions or individuals for the purpose of scholarly research.

I understand that my thesis may be made electronically available to the public. 


\begin{abstract}
Analysis of Electrooculogram (EOG) Signals in Studying Myasthenia Gravis
\end{abstract}

\author{
Master of Applied Science, 2017 \\ Timothy Liang \\ Electrical and Computer Engineering \\ Ryerson University
}

Myasthenia Gravis (MG) is a neuromuscular disorder that induces muscle weakness and fatigue which can be fatal. A common precursor for severe form of MG is ocular MG. In this thesis, we explored signal processing methodologies for early stage detection of MG using electrooculogram (EOG) signals.

An EOG signal database consisting of 62 control and $16 \mathrm{MG}$ (mild to moderate) subjects were analyzed for eye movement characteristics and EOG signal morphologies using time domain and wavelet domain techniques. A linear discriminant analysis (LDA) based classifier was used to quantify the ability of features in separating MG from control samples. Average overall classification accuracy achieved by the proposed method for the best time domain feature (average rise rate) and best wavelet feature (scale band energy) was $82.5 \%(\mathrm{P}<0.01, \mathrm{AUC}=0.887)$ and $83.8 \%$ $(\mathrm{P}<0.01, \mathrm{AUC}=0.893)$, respectively. The obtained results suggest $\mathrm{EOG}$ based analysis is a viable, non-invasive alternative MG screening method. 


\section{Acknowledgment}

I acknowledge my supervisor Dr. K. Umapathy for his guidance and motivation. I would also like to thank my clinical co-supervisor Dr. M. Boulos from Sunnybrook Hospital and our research collaborator Dr. H. Katzberg from Toronto General Hospital for their valuable clinical input and providing the data for this work. I would like to extend my thanks to my parents, sister, and my friend Albert Ngu for their support. 


\section{Contents}

$\begin{array}{ll}\text { Abstract } & \text { iii }\end{array}$

List of Tables

List of Figures

1 Introduction 1

1.1 Myasthenia Gravis . . . . . . . . . . . . . . . . . . . . 1

1.2 Diagnosis Methods . . . . . . . . . . . . . . . . . . 5

1.3 Motivation . . . . . . . . . . . . . . . . 7

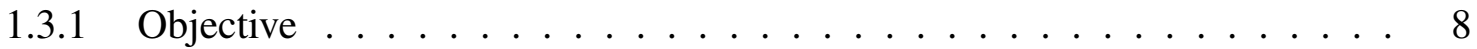

1.4 Thesis Outline . . . . . . . . . . . . . . . . . . . . . . . . . . 9

2 Background $\quad 11$

2.1 Electrooculogram . . . . . . . . . . . . . . . . . . 11

2.2 Clinical Sleep Test . . . . . . . . . . . . . . . . . . . 13

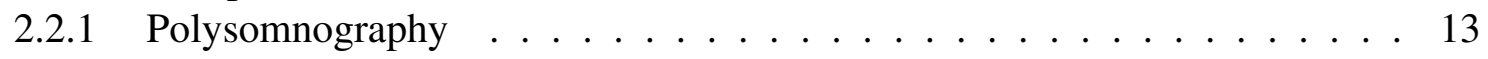

2.2.2 Multiple Sleep Latency Test . . . . . . . . . . . . . . . . . . . . . . . 13

2.2.3 Maintenance of Wakefulness Test . . . . . . . . . . . . . 15

2.3 Existing Literature . . . . . . . . . . . . . . . . . . . . . . . . . . . . . . . . . . . .

2.4 Time Domain Movement Detection . . . . . . . . . . . . . . . . 17

2.5 Wavelet Transform . . . . . . . . . . . . . . . . . . . . . . 19

2.6 Pattern Classification . . . . . . . . . . . . . . . . . . . . . . . . . . . . . . . . . .

2.6.1 Fisher's Linear Discriminant Analysis . . . . . . . . . . . . . . . . . . 24

2.7 Chapter Summary . . . . . . . . . . . . . . . . . . . . . . . . . . . . 29

3 Time Domain Analysis $\quad 30$

3.1 Time Domain Eye Movement Feature Analysis . . . . . . . . . . . . . . . . . 30

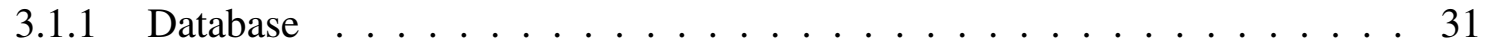

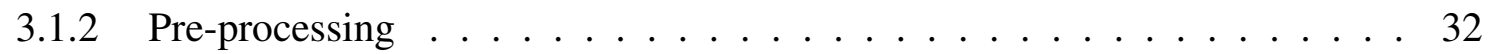

3.1.3 Eye Movement Detection Algorithm . . . . . . . . . . . . . . . 34

3.2 Feature Extraction . . . . . . . . . . . . . . . . . . . . 39

3.2.1 Average Horizontal Slow Eye Movement . . . . . . . . . . . . . . . 39 
3.2.2 Average Rise and Fall Rate . . . . . . . . . . . . . . . . . . . . . . . 40

3.3 Feature Selection . . . . . . . . . . . . . . . . . . . . 41

3.4 Results and Discussions . . . . . . . . . . . . . . . . . . . . 41

3.4 .1 Group All . . . . . . . . . . . . . . . . . . . 42

3.4 .2 Group MSLT . . . . . . . . . . . . . . . . . . . . . . . . 44

3.4 .3 Group PSG . . . . . . . . . . . . . . . . . . . 44

3.5 Chapter Summary . . . . . . . . . . . . . . . . . . . . . . . . 47 47

4 Wavelet Domain Analysis $\quad 48$

4.1 Wavelet Analysis . . . . . . . . . . . . . . . . . . . . . . 48

4.1 .1 Wavelet Selection . . . . . . . . . . . . . . . . . . . . . . . 49

4.1 .2 Scale Selection . . . . . . . . . . . . . . . . . . 51

4.2 Feature Extraction . . . . . . . . . . . . . . . . . . . . 52

$4.2 .1 \quad$ Scale Band Energy . . . . . . . . . . . . . . . . . . . . . 52

4.2 .2 Scale Distribution Width $\ldots \ldots \ldots \ldots$

4.3 Feature Selection . . . . . . . . . . . . . . . . . . . . . . 54

4.4 Results and Discussion . . . . . . . . . . . . . . . . . . 54

4.4 .1 Group All . . . . . . . . . . . . . . . . . . 54

4.4 .2 Group MSLT . . . . . . . . . . . . . . . . . 56

4.4 .3 Group PSG . . . . . . . . . . . . . . . . . 57

4.5 Chapter Summary . . . . . . . . . . . . . . . . . . . . . . . . 59

5 Conclusions and Future Works $\quad 63$

$5.1 \quad$ Summary of Results . . . . . . . . . . . . . . . . . . . . . . . . . . 64

5.2 Potential Applications . . . . . . . . . . . . . . . . . . . . 65

5.3 Directions for Future Works $\ldots \ldots \ldots 6$

$\begin{array}{ll}\text { Bibliography } & 67\end{array}$ 


\section{List of Tables}

3.1 LOO cross validated classification accuracy using Rise Rate for Group All. . . . . 42

3.2 LOO cross validated classification accuracy using Rise Rate for Group MSLT. . . . 44

3.3 LOO cross validated classification accuracy using Rise Rate for Group PSG. . . . . 46

4.1 LOO cross validated classification accuracy using Group All with SBE mean feature (Mexican Hat Wavelet). . . . . . . . . . . . . . . . . . 56

4.2 LOO cross validated classification accuracy using Group MSLT with SBE mean feature (Mexican Hat Wavelet). . . . . . . . . . . . . . . . . . . . 57

4.3 LOO cross validated classification accuracy using Group PSG with mean SBE feature (Mexican Hat Wavelet). . . . . . . . . . . . . . . . . . 57

4.4 LOO cross validated classification accuracy using Group All with mean SBE feature (Morlet Wavelet). . . . . . . . . . . . . . . . . . . . . . . 60

4.5 LOO cross validated classification accuracy using Group MSLT with mean SBE feature (Morlet Wavelet). . . . . . . . . . . . . . . . . . . . . 60

4.6 LOO cross validated classification accuracy using Group PSG with mean SBE feature (Morlet Wavelet). . . . . . . . . . . . . . . . . . . . . . 60

4.7 LOO cross validated classification accuracy using Group All with mean SBE feature (Gaussian 2 Wavelet). . . . . . . . . . . . . . . . . . . . . . 61

4.8 LOO cross validated classification accuracy using Group MSLT with mean SBE feature (Gaussian 2 Wavelet). . . . . . . . . . . . . . . . . . . . . . 61

4.9 LOO cross validated classification accuracy using Group PSG with mean SBE feature (Gaussian 2 Wavelet). . . . . . . . . . . . . . . . . . . 61 


\section{List of Figures}

1.1 Example of generic symptoms for patients who have ocular weakness. . . . . . . 3

1.2 Sample waveform showing EOG signals from the left eye channel (top) and right eye channel (bottom). . . . . . . . . . . . . . . . . . . . 7

1.3 Block diagram of the overall thesis. . . . . . . . . . . . . . . . . . . . 9

2.1 Illustration of how signal profiles are formed with respect to the EOG channel. . . 12

2.2 Front view of electrode setup used to acquire EOG signals. . . . . . . . . . . . . 14

2.3 Top view of electrode setup used to acquire EOG signals. . . . . . . . . . . . . . . 14

2.4 Example of a sample scalogram with continuous energy distribution. . . . . . . . . 20

2.5 Sample ROC curve. . . . . . . . . . . . . . . . . . . . . . . . . . . . . 29

3.1 Block diagram showing the overview of chapter 3 . . . . . . . . . . . . . . 31

3.2 Block diagram showing the processes involved in the pre-processing procedure. . . 33

3.3 Block diagram displaying the process of the eye movement detection algorithm. . . 36

3.4 Illustration of eye movement detection algorithm using a sample EOG signal. . . . 37

3.5 Sample LOC and ROC EOG signal illustrating anti-phase. . . . . . . . . . . . 38

3.6 Algorithm for detecting genuine horizontal movements. . . . . . . . . . . . . . 38

3.7 Boxplot figures for each group and feature. Each column represents a group while each row represents a feature. . . . . . . . . . . . . . . . . . . 43

3.8 ROC curve for classifier using rise rate for Group All. . . . . . . . . . . . . . . . 45

3.9 ROC curve for classifier using rise rate for Group MSLT . . . . . . . . . . . . . . 45

3.10 ROC curve for classifier using rise rate for Group PSG. . . . . . . . . . . . . . . 45

4.1 Block diagram showing the overview of chapter 4 . . . . . . . . . . . . . . . . 49

4.2 Sample MG EOG morphology compared to different wavelet structures. . . . . . . 50

4.3 Boxplot results for each group using the SBE feature from LOC, ROC, and Mean of the two channels. . . . . . . . . . . . . . . . . . . . . . . . . . 55

4.4 ROC curve for classifier using mean SBE for Group All . . . . . . . . . . . . . 58

4.5 ROC curve for classifier using mean SBE for Group MSLT. . . . . . . . . . . . . . 58

4.6 ROC curve for classifier using mean SBE for Group PSG. . . . . . . . . . . . . . . 58 


\section{Chapter 1}

\section{Introduction}

$\mathrm{M}$

UCLES are vital to the functional integrity of the human biological system. Fundamental

tasks for survival such as breathing is controlled by the contraction and expansion of the diaphragm muscles. When these muscles from the vital organ group are affected by neuromuscular disorders, it could lead to fatal conditions. Myasthenia gravis (MG) is one such neuromuscular disorder that induces muscle fatigue and weakness. Once the disorder develops in vital muscle groups of the body, such as muscles that aid breathing or swallowing, could possibly prove to be fatal. Currently, such critical conditions are managed in intensive care units and in turn, reduces the mortality rate for these patients [1]. To have a more complete understanding of MG, the disease's nature, current treatment options and available diagnostic approaches needs to be further explored. With the current approaches alone, early identification of MG in patients is difficult. Hence, requires attention to develop new methodologies to assist prevention strategies and appropriate treatment.

\subsection{Myasthenia Gravis}

Clinically, MG is characterized as painless, gradual weakening of striated muscle over repetitive or continued activity. However, the weak condition of the muscle will improve with sufficient rest [2]. MG affects the neuromuscular junction site, which is a location for the nerve fiber and muscle cell to communicate. At these locations, certain enzymes are present and are needed to establish communication between the nerve fiber and muscle. With MG, the acetylcholine substance is attacked 
by anti-acetylcholine receptors, which leads to the decrease in muscle activity. Another source that could contribute to the manifestation of MG is the anti-muscle-specific tyrosine kinase (antiMuSK), which also impacts the neuromuscular junction site [3,4]. The MuSK receptor is required for maintaining the neuromuscular junction as well as its formation during fetal development [5]. The MG disorder could affect different muscle groups such as ocular and limbs. Depending on the location of where the disease is present, it determines the severity of the condition.

The MG disorder is not limited to one single form. This disorder progresses over time and the different forms are dictated by the level of severity, onset period, and localization of the neuromuscular junction failure. A brief classification of MG is listed as [6]:

- Early-onset (before the age of 50)

- Late-onset (after the age of 50)

- Thymoma-related

- MG with the presence of anti-MuSK antibodies

- Ocular MG

- MG without detectable acetylcholine receptors and MuSK antibodies

If the forms of myasthenia gravis is localized in the ocular region of the body, then it is referred to as ocular myasthenia gravis (OMG). For MG to be fully categorized as OMG, the distribution of MG symptoms must be limited to the ocular region for over 2 years $[1,7]$. This form could be seen as an early precursor to more severe forms of generalized MG (GMG). Up to approximately $65 \%$ of MG patients show signs of ptosis or weakness in the extraocular muscles [8]. An illustration of how ocular weakness appears in patients is shown in Figure 1.1. Within the group of patients exhibiting symptoms from the ocular area, $50-80 \%$ of them will develop into the generalized form $[9,10]$. Majority of MG patients with ocular symptoms will progress to GMG within the 2 year period after the symptoms first appear [11]. Based on these reported statistics, the presence of ocular symptoms indicates a fair possibility of developing from mild MG into more severe forms of 


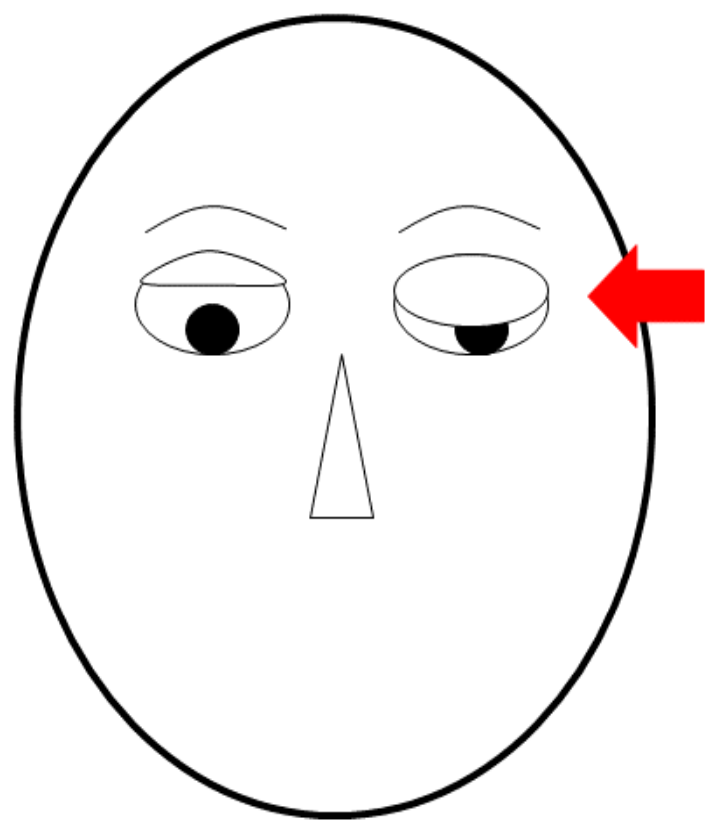

Figure 1.1: Example of generic symptoms for patients who have ocular weakness.

GMG. Thus, the ocular muscles are an important region to focus on as it potentially provides early indication linking to the $\mathrm{MG}$ disorder.

Onset of MG occurs at different ages and is associated to different gender groups. For ages 50 and under, females were more commonly affected in comparison to males, but the opposite trend occurs between 60 to 80 years of age [2]. Nonetheless, MG is able to affect a person at any age. Depending on the region, one gender may be more susceptible to the other. In a review, males appear to be affected more commonly than females in India [9]. The progression of MG changes over time and observing a compilation of previous case studies, incidence and prevalence changes with respect to the demographic. According to a variety of works from Europe, incidence and prevalence varies between 6.3-28.0 per million and 70.6-328.9 per million, respectively [12]. In a relatively recent study [13], the prevalence and incidence of MG of studied in Ontario, Canada over the years of 1996-2013. It was found that the incidence between the specified years remain practically the same, but the prevalence displayed an increase. Over the course of the 17 year period, it appears that the crude prevalence almost doubled from 1996 and 2013. The crude incidence and prevalence of MG cases in Ontario as of the year 2013 were found to be 2.8 per 100,000 
population and 32.0 per 100,000 population. It was also observed that during this timespan, the prevalence between males and females steadily showed less difference.

The medical treatment of of MG varies depending on the condition of the disorder that the patient was diagnosed with. Some common treatment approaches include the use of symptomatic drug therapy, immunosuppressive drug therapy, thymectomy and supportive therapy [14]. The use of immunosuppressants and thymectomy could be avoided if the patient's MG condition remains stable [15]. These two approaches are for more severe cases of MG as both these treatment plans have drawbacks. Using immunosuppressants allows the body to become more susceptible to lifethreatening infections and other diseases. In regards to thymectomy, it is a surgical procedure in the removal of the thymus. The removal of the thymus, according to a report, was accepted as a form of treatment as it demonstrated relief from MG symptoms [16]. However, the success of the treatment method is dependent on the technique used and usually, the results of thymectomy are not immediate and will not be seen until after a few months or years [17]. If the patient is in a more severe stage of MG, then intensive care treatment is required. Generally, this therapy option is administered when the patient shows weakness in their respiratory muscles and the symptoms intensify.

With drug therapy, dosage levels and types of drugs will be adjusted corresponding to the patient's symptoms. For MG characteristics that are not life-threatening, such as ocular symptoms, drugs containing acetylcholinesterase inhibitors (AChEIs) are sufficient. For more generalized MG, immune response adjustment treatments are also required [18]. Intentions for using medication containing AChEIs is to inhibit or prevent the acetylcholinesterase enzyme from decomposing acetylcholine. The use of steroids may be needed when AChEIs are no longer enough to suppress the MG symptoms $[19,20]$. However, the quality of life in short or long terms will be affected with the use of steroids as the dosage levels required are high. Typically, with high dosages of steroids or subcategories of steroids, such as usage of corticosteroids for early stage MG treatment, leads to other undesirable effects. Possible side effects of using corticosteroids include bone degeneration, diabetes, hypertension, osteoporosis, and many more diseases and disorders [7]. Due to the diverse subclassification of $\mathrm{MG}$, no treatment is more superior to the other. As the risks from treatments 
vary according to the severity of MG, prescribing the treatment at earlier stages would reduce the degree of side effects to the patient. To determine mild or early stages of MG, focusing on common indicators, such as ocular symptoms, could prove to be significantly useful. Thus, with the disorder detected earlier, the treatment options could then be properly optimized to increase the overall effectiveness of the therapy.

\subsection{Diagnosis Methods}

As mentioned previously, the MG disorder could become a nuisance to a person's quality of life if the disease is not treated or found in a timely manner. All treatment plans are only useful if the disease is detected correctly. Over time, various procedures or techniques with or without technological aid were developed for clinicians to diagnose MG. Currently, the electrophysiological methods to diagnose MG are single-fiber electromyography (SFEMG) and repetitive nerve stimulation (RNS). The two approaches have their respective benefits and drawbacks.

The SFEMG method is highly sensitive in detecting abnormalities in neuromuscular transmission [9]. In SFEMG, a fine needle is used to perform the acquisition. It records the difference in conduction times between individual muscle fibers. Although the SFEMG approach provides high sensitivity in the detection of neuromuscular defect, the drawback of this method is that it is unable to distinguish between other neuromuscular abnormalities that may be present when performing the diagnosis. In other words, the SFEMG is able to detect MG, but it cannot state whether or not MG is the only disorder present $[21,22]$. Comparing the more common initial form of MG with its developed form, the sensitivity range differs between the two. It is mentioned that the sensitivity range is less in the ocular form in comparison to the generalized [9]. For the purpose of early detec-

tion of MG, SFEMG would have difficulties in achieving that objective due to reduced sensitivity and lack of indication for MG specifically. If the MG disorder is more evident in the patient or the dysfunction at the neuromuscular junction is higher, then the use of SFEMG would be ideal for detection. Another disadvantage to consider is the operation of the SFEMG itself. Since the device requires a trained expert to operate the device, its usage becomes limited [23,24], especially for screening a large group of patients. Thus, the accessibility to this diagnostic approach for patients 
becomes more scarce.

In comparison to the SFEMG approach, the RNS method uses surface electrodes to transmit pulses and cause stimulation. On top of being non-invasive, it is also commonly regarded as the fastest first stage of diagnosis for MG patients [23]. The overall RNS approach proceeds by stimulating the muscle with a pulse at a particular frequency and the muscle action potentials are recorded. From the measured potentials, a decrease in response is observed over time. However, according to literature, the sensitivity ranges will vary and relies on the muscle of interest and decremental threshold value $[24,25]$. Comparing diagnostic performance with SFEMG, RNS does fall short in terms of sensitivity for diagnosing MG. The sensitivity of RNS decreases and is also lower in mild cases, such as ocular. Initial examination studies still prefer the use of RNS because it provides higher specificity and availability in contrast to SFEMG.

Aside from the electrophysiological diagnostic approaches mentioned for MG, there are other studies that use alternate signal acquisition methods to detect the disorder. Previous attempts to study MG, specifically OMG, were shown in a comparative study exploring other acquisition techniques such as electrooculograms (EOG), search coils, and infrared [26]. In a more recent work [27], OMG diagnosis was performed on using ocular vestibular evoked myogenic potentials (oVEMP). The oVEMP is used to record the activities of the inferior oblique eye muscle and the decrement in signal amplitude is related to fatigue. As for this thesis, EOG signals will be used because it extracts biosignals, which represent an individual's eye movement localized in the whole ocular region rather than the localized inferior oblique eye muscle. This signal acquisition method could provide useful information for early detection as it observes the ocular behaviour of the patient, which could potentially capture the ocular weakness symptoms. In comparison to the current methods of MG detection, it is also a non-invasive procedure and causes minimal discomfort. A sample of EOG signals generated from the left and right channel is shown in Figure 1.2. Movements of the eye are only possible due to the contraction of the ocular muscles. Referring to the clinical definition of MG and its symptoms that were previously established, the eye movements could show possible deviations due to the weakness and weariness of the ocular muscles as they are early symptoms of MG. The difference between this work and any previous attempts 

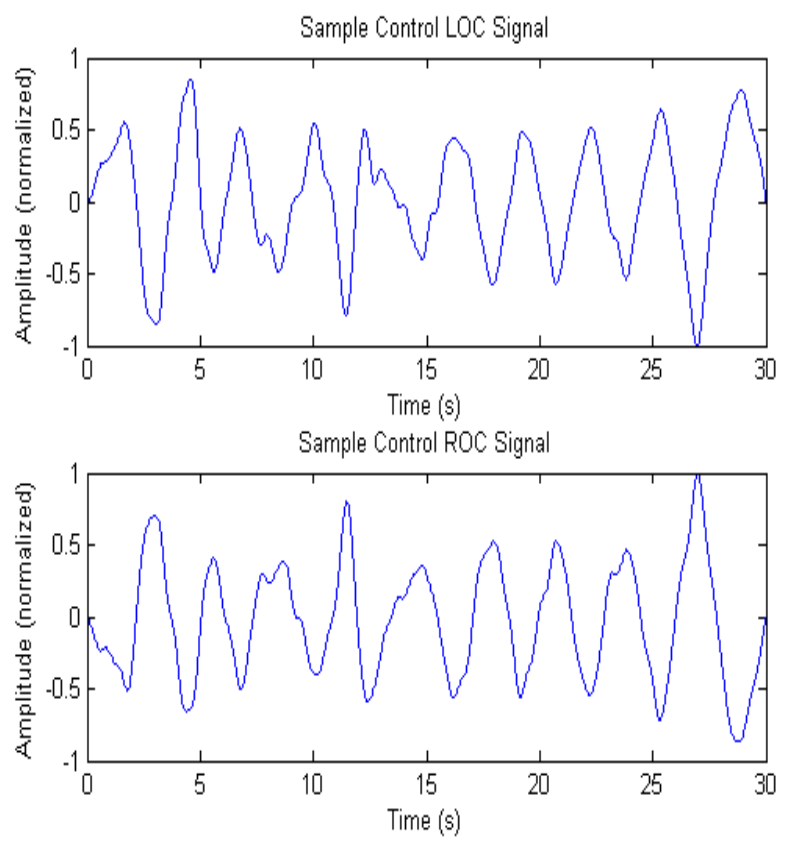

Figure 1.2: Sample waveform showing EOG signals from the left eye channel (top) and right eye channel (bottom).

with EOG (MG or otherwise) is the setting or condition the patient was in before the acquisition process. Patient data was recorded from different clinical sleep tests; each test requiring different preconditions. The basics of EOG and their propagation will be explained in greater detail later on in Chapter 2.

\subsection{Motivation}

As mentioned previously, the MG disorder could hinder a person's well-being if the disease is not treated or found in a timely manner. All treatment plans are only useful if the disease is detected correctly. Among the electrophysiological techniques, currently the methods to diagnose MG are SFEMG and RNS. The two approaches have their respective advantages and disadvantages. While SFEMG is superior in terms of detection sensitivity, RNS compensates for its reduced speed and non-invasiveness. These current methods are more suitable for detecting the developed form of MG. However, early detection of MG is important as it could help clinicians select more optimal treatments and prevent the emergence of more severe MG. Common traits in early stages of MG 
were observed to be muscular weakness and fatigue in the ocular site.

Hence, if EOG signals are able to detect ocular symptoms caused by MG in a non-invasive manner, it could lead to further development of early detection for MG. As it stands, both RNS and SFEMG alone is not sufficient enough in diagnosing early stages of MG. EOG could provide a better means in quantifying ocular symptoms of MG in mild forms as it extracts information in the ocular region. Although RNS also uses surface electrodes, the approach is technically noninvasive, but is intrusive in this application. Since the technique transmits a stimulating pulse to the patient, it disturbs the natural state of the pathology and the body's response. In comparison, EOG is passive as it does not agitate the body with any form of electrical stimulation and does not penetrate the body. Consequently, EOG will be able to observe the body's response under the effects of MG more naturally. The ability to detect MG early based on solely on its early stage symptoms will lead to more prompt diagnosis and proper treatment to avoid the progression of MG of into a lethal condition.

\subsubsection{Objective}

The objective of this work is to explore the possibility of detecting early stage MG by analyzing EOG signals. The EOG signals used in this study were extracted from patients participating in different clinical sleep tests, each requiring a different precondition. Each of these sleep tests and their respective protocols will be further discussed in Chapter 2. By applying different signal processing techniques, a variety of features could then be extracted and grouped. Depending on the outcome of the classification, it will determine if the use of EOG signals is a viable noninvasive alternative method for the early detection of MG. As mentioned, there are existing signal acquisition techniques for detecting MG, but each one has their respective limitations. The current study emphasizes more on the viability of the features extracted from sleep test EOG signals. The patients used for the analysis were already examined by physicians and determined to have MG (mild to moderate). The result of this work could also determine the potential of using sleep test-based EOG signals as indicators for patients with the disorder.

To observe the viability of using EOG signals, two approaches were attempted and their re- 


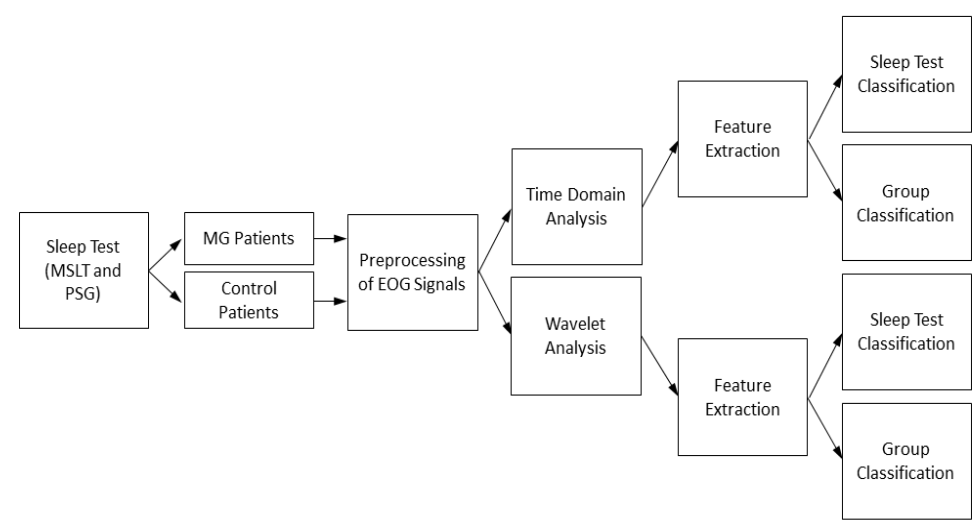

Figure 1.3: Block diagram of the overall thesis.

spective results were used to compare performance. The process of this work can be divided into three stages. First, the raw EOG signals are preprocessed for more consistent analysis between patient groups and sleep tests. Clinical sleep testings used in this study were the multiple sleep latency test (MSLT) and polysomnography (PSG). The individual EOG signals were then divided into smaller segments to further simplify the analysis process. Then, the work is separated into two, each applying a different signal processing methodology. The time domain analysis was first attempted by exploring the existing, well-known time domain eye movement detection methods. This process will validate the possibility of using EOG signals and serve as a reference for MG and control separation. Afterward, the wavelet analysis approach on the same set of preprocessed signals were attempted to observe the signal's characteristics in the time-frequency or time-scale domain. The wavelet approach may provide features to identify morphological structures in better understanding MG. The overall outline of the thesis is illustrated as a block diagram in Figure 1.3.

\subsection{Thesis Outline}

The thesis is subdivided into different chapters with sections and subsections for different stages of this research study. It is partitioned as described below: 
- Chapter 2: This chapter will explain the signal of interest and how it is generated. Each of the clinical sleep tests used will then be defined and elaborated. Existing, common time domain features will be explored in terms of their extraction procedure and application. Afterward, the chapter will cover the background material of signal processing methods used in this work. Lastly, the chapter will explain the pattern classification scheme implemented in this thesis.

- Chapter 3: This chapter contains the proposed time domain methodology of differentiating the MG group from the control group. It will discuss the process of the time domain algorithm, applied pre-processing methods, features extracted for classification, and results obtained from this approach.

- Chapter 4: This chapter contains the proposed time-frequency domain approach to the problem. The motivation for using this method will be discussed along with the different combinations of features extracted from the generated algorithms. The obtained results based on different sleep tests, selection of features, parameters chosen, and selected wavelets will be examined.

- Chapter 5: This chapter summarizes the overall thesis as well as provides more in depth discussions regarding the study. It will consist of the potential of the work, its future direction, and conclusion based on the achieved results. 


\section{Chapter 2}

\section{Background}

7 HIS chapter's focus will be on outlining the required signal processing theory for analyzing

1 EOG signals. The chapter will begin with describing EOG and its acquisition process. It then proceeds to cover the time domain based analysis of EOGs and existing methods of eye movement detection found in literature. The time domain methods used will be discussed more in depth, mainly focusing on how these eye movement features were generated. Subsequently, the time-frequency domain approach and its relevant theory will be presented. The chapter will then explain the pattern classification, its importance, and which method of classification was used for this study.

\subsection{Electrooculogram}

The EOG is a waveform that is generated by the potential difference from the cornea and retina. This cornea-retina potential may be considered as an electrical dipole, where the cornea or the front of the eye is the positive end and the retina is the negative [28]. By observing the EOG, the signal can determine the position and direction of the eye. The eye movements could be later determined over a period of time by the change in polarity of the waveform. Typical amplitude values of EOG ranges from 50 to $3500 \mu \mathrm{V}$ with a frequency range of about $\mathrm{DC}-100 \mathrm{~Hz}[29,30]$.

The electrodes are labeled according to its respective location: right channel (ROC), left channel (LOC), and vertical channel (VOC), as shown in Figure 2.1. When the eyes move towards the electrode, it will be detected and generated as a positive peak and negative for the reverse action. 

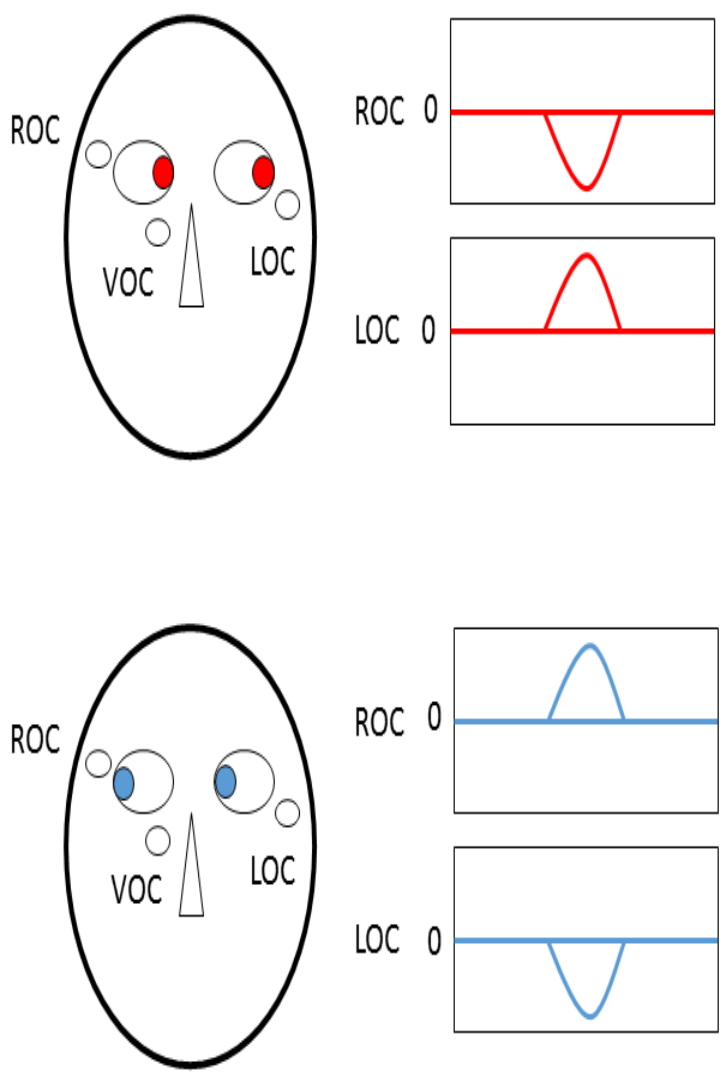

Figure 2.1: Illustration of how signal profiles are formed with respect to the EOG channel.

To observe the wave generation visually, the signal profile is illustrated in Figure 2.1 when the person moves their eyes to the left (red signal) and right (blue signal). As the eyes move to the left, the LOC produces a positive peak while the ROC produces a negative. An opposite effect is seen when the eyes are shifted to right. The phenomenon where the signals appear anti-phase could be related to Hering's law. The definition of Hering's law is that eyes are arranged mirrorsymmetrically for neural and muscular control of both the right and left eye, the movement of the two eyes is normally parallel [31]. Keeping this law in mind, it serves as a reference to determine what type of eye movements could be considered as normal.

The generation of EOG signals is relative to the positioning of the surface electrode. The general electrode setup used is shown in Figure 2.2 and the electrode placement for the reference 
points are shown in Figure 2.3. Although each channel will generate a signal based on the eye's movement, the difference between the channel and a reference point furthest away was taken. For example, the LOC will refer to A2 while the ROC and VOC refers to A1.

\subsection{Clinical Sleep Test}

For this study, EOG signals were generated from three different clinical sleep tests: polysomnography (PSG), multiple sleep latency test (MSLT), and maintenance of wakefulness test (MWT). The three sleep tests were considered because each sleep test examines a different sleep trait from an individual. To perform each test, a different precondition was required.

\subsubsection{Polysomnography}

The PSG is a sleep test that is performed overnight to observe the sleeping and breathing patterns of the subject. To evaluate sleep, different physiological parameters from the individual are extracted. Aside from EOG signals, the PSG also provides other physiological information such as EEG, EMG, respiratory, and ECG. Depending on the sleep disorder of interest, not all physiological channels are required for the sleep study [32]. The PSG sleep test has been used to help diagnose various disorders. Some of the more common uses of PSG tests were for sleep apnea, which is a situation where the individual faces difficulties breathing during sleep. For conditions such as sleep apnea, the PSG sleep test is considered a gold standard diagnostic test [33]. Other applications where PSG has been applied extends to neuromuscular disease. The definition of MG was described as a neuromuscular disorder and is able to affect different muscle regions on the body. Thus, observing physiological signals extracted from the PSG test could provide insight to the neuromuscular activities that are occurring in the body. For the intentions of early detection in this study, we will focus on ocular activity as reflected on the EOG signals.

\subsubsection{Multiple Sleep Latency Test}

The MSLT is an objective measure to examine the ability of falling asleep under the conditions that are absent of external stimulating sources. Results from this sleep test will indicate the sleepiness of 


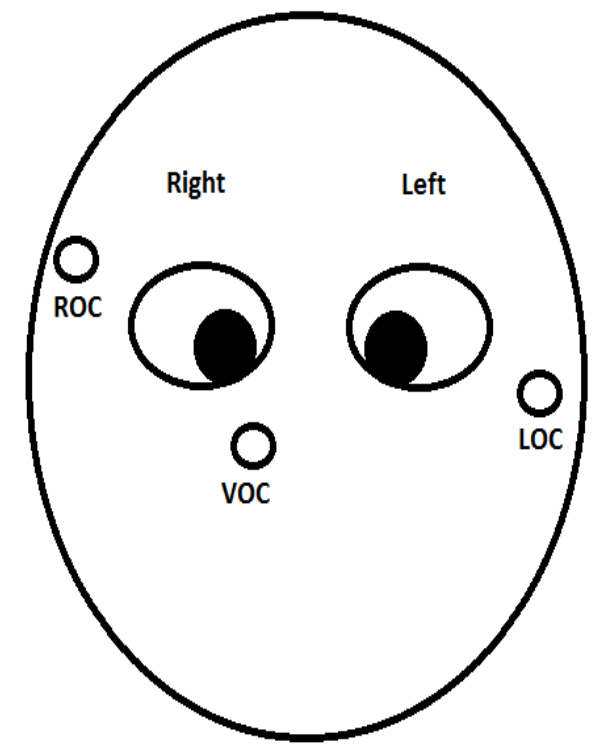

Figure 2.2: Front view of electrode setup used to acquire EOG signals.

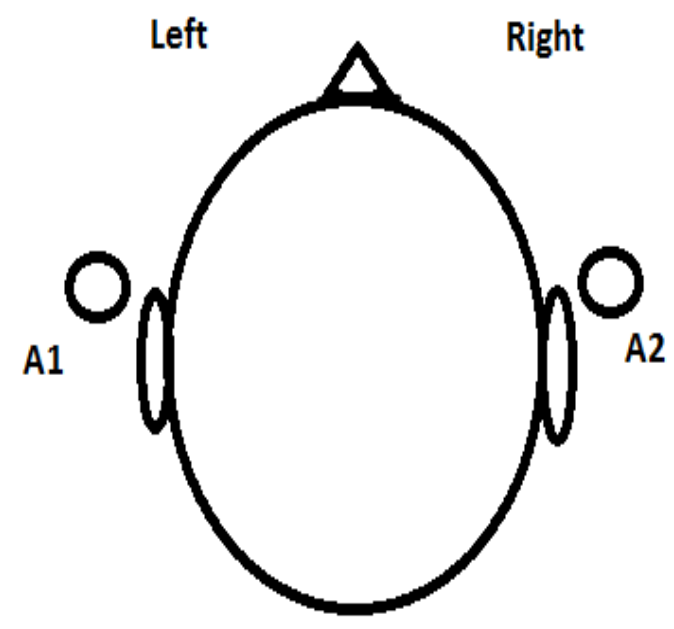

Figure 2.3: Top view of electrode setup used to acquire EOG signals. 
the individual derived from their sleep latency. This form of sleep test does demonstrate reliability as its results are not affected by reiteration interval or by the degree of sleepiness [34]. To conduct the sleep test, the protocol is to initiate the test after the subject has had at least 6 hours of rest. Thus, this test is taken sequentially after an overnight PSG testing. However, the subject's sleep result can be affected by previous sleep-wake cycles up to 7 days. For standardization purposes, the subject is stationed in a dark and quiet room, at temperature levels comfortable for the patient. The subject is instructed to assume a comfortable position and attempt to fall asleep. For ideal results, the subject should be off any stimulating and rapid eye movement (REM) suppressing medications for two weeks before testing [35]. The MSLT is useful in aiding the diagnosis of sleep disorders such as narcolepsy. Although the application is different, the MSLT may prove to be useful for early MG detection as it observes the fatigue in eye movements. From the literature survey [8], common traits of early stage MG are ocular weakness and fatigue. Thus, MSLT recordings may provide useful insight in separating the MG group and potentially be used for early MG detection.

\subsubsection{Maintenance of Wakefulness Test}

The MWT is an objective measure that observes the individual's ability to stay awake for a given duration. For this sleep test, prior sleep tests, such as a PSG, and sleep logs are not required and is decided by the clinician [34]. To perform the test, it is recommended to be conducted 90 minutes to three hours after waking up. The test is divided into 4 trials with 2 hours intervals in between. The patient is stationed in a room that has sufficient amount of external light and just out of the patient's field of vision. As the patient is seated on a bed, their head and back are supported such that no discomfort is felt by the neck due to flexing or extending. The MWT is useful for obstructive sleep apnea, narcolepsy, and potentially other sleep disorders. For occupations or tasks that are highly dependent on the ability to remain awake, the MWT assessment may be used for evaluation. This sleep test was not used in our study due to the lack of matching data. The description provided here is for the sake of completion. 


\subsection{Existing Literature}

After understanding how and where these EOG signals are generated from, existing works regarding eye movement analysis have been examined. Specifically for EOG based eye movement detection, an array of eye movement features were studied. Understanding some existing works that applied time domain only or time-frequency domain approaches will provide insight on what is viable or carries considerable potential. EOG signals have many uses which consist of: eyebased control systems, medical applications, or automated processes such as classification for sleep stages.

Different applications for eye-based control systems have been attempted. There are works that uses eye movements as a control mechanism to interface with devices. In a study conducted by Barea et el. [36], their focus was on enabling patients with disabilities to control a wheelchair using eye movements. Gaze angle was quantized based on the amplitude of the EOG signals at measured angles and the direction was decided based on the polarity of the signal from each channel. A similar work [37] was done, but used a time-frequency approach to extract energy coefficients at different frequency ranges. Each basic eye movement (up, down, left, and right) was then paired with the dominant energy scale or frequency range for the purpose of wheelchair control for paralyzed patients. In another work [38], their focus was to use EOG signals for the purpose of human computer interface. Horizontal and vertical eye movements were used to select letters, and these motions were then measured.

The use of EOG signals and eye movements could be applied for medical applications. It could be for monitoring ocular behaviour for various purposes such as progression of disorders or effects of medication. In one study [39], early diagnosis for MG was attempted with the use of saccadic eye movements. Different waveforms were categorized as certain movements and were used to classify the MG patients as purely extraocular, purely ocular, or both extraocular and ocular. Use of EOG and eye movement information to aid diagnosis also expands to other eye related diseases, such as Retinitis Pigmentosa [40] and Best's disease [41]. Aside from diagnostic applications, eye movements could also be used for observing the effects of medication [42]. The study applied eye movement detection by detecting local maxima and minima obtained from three EOG channels. 
Eye movement directions were then determined based on the combinations of local maxima and minima.

Other clinical uses would be tracking eye movements under the effects of medication. In one study [43], the eye movements were separated based on certain frequency ranges. The EOG signals were filtered with the discrete Fourier transform and the inverse discrete Fourier transform was taken for two specified frequency ranges. Coupled with cross-correlation, peak-to-peak amplitude difference, and thresholds, the different stages of sleep were classified. Another method to classify the sleep stages was using EOG signals in conjunction of EEG and EMG signals, and classification was with the use of artificial neural networks [44]. In this thesis, the sleep stages were segmented in time with the aid of a hypnogram. The hypnogram is a universal method for assessing sleep stages, and represents the labeling of sleep stages performed by an expert [45].

\subsection{Time Domain Movement Detection}

There are different methods of extracting information relating to different aspects of motions relating to the eye. Depending on which eye movement feature is of interest, the horizontal (left and right), vertical (up and down), or combination of the two movement directions could be used. Some of the common features used in previous studies include blink, or saccades. Saccades refers to the movement of both eyes, which could vary in speeds and duration. To subdivide saccade movements further, it could also be labeled fast or slow. These commonly found features are explored and explained on how they were derived.

One common feature used in time domain was calculating the horizontal movements. These horizontal movements are generated by combining the left and right channels. Other features that provide useful information for analysis are slow eye movements. If a single movement exists over a duration of time exceeding a certain threshold, it can be categorized as a slow eye movement (SEM) and be applied to saccadic movements. This feature is commonly used due to studies suggesting that SEM are indicators to delayed response. In a previous work [46], using EOG signals, the SEMs were derived from forming the horizontal movements of the eye and extracted the amplitude and mean velocity. Based on a threshold, the eye movement was judged as either 
slow or not. The use of a threshold to determine movements was also applied in a work done by Boulos et al. [47]. Two thresholds were applied in this work, one for validating a movement and a secondary threshold to classify whether the movement was fast or slow.

Considering the nature of a normal blink, it will cause a change in the EOG waveform. To characterize this eye movement, some works use either EOG signals measured from the vertical channel or in conjunction with the horizontal. In a work done by Bulling et al. [48], the EOG signal from the vertical channel was used and blinks were considered as two large peaks in succession, one positive and one negative. Understanding that the time delay between the sequential positive and negative peak was lower than two saccadic movements, a threshold was used to classify them. In another work [49], the vertical and horizontal channel were used to detect blink. For a normal blink, the vertical channel would detect a positive pulse while the horizontal channel remains relatively zero. This work also took blink eye movements further by examining other actions with blinks, such as double blinks and winks. The decision process was to examine the amplitude from the vertical and horizontal channels, and the maximum cross correlation between the EOG signal from the vertical channel and a template signal exhibiting double blink.

The time domain approach performs adequately with the use of eye movement features. However, according to the understanding of MG of its clinical characteristics, the time domain approach is not sufficient for fully detecting the changes from MG patients. As previously discussed, the MG disorder is able to progress in its symptoms and intensity. Increase in muscle weakness and fatigue in the affected region will cause a change in the muscle activity. The electrophysiological representation and nature of MG would then alter with time, resulting in a change in the signal's structure. The application of time-frequency signal processing techniques are then required to obtain a more thorough analysis of morphological structure. Thus, the next stage of the thesis will focus on the theory and application of time-frequency domain approach to determine if the morphological characteristic can be captured. 


\subsection{Wavelet Transform}

As the name implies, the wavelet transform makes use of the smaller waves or wavelets to correlate with the signal of interest. These wavelets are mathematical expressions that consist of certain properties and have definitive time-frequency localization. Based on different mother wavelets, different aspects or characteristics of the signal in the time-frequency domain could then be seen. As different variations of mother wavelets could be propagated through scaling and translating, this results in a highly flexible tool for a wide range of applications. Characteristics of the mother wavelet would dictate the nature of the application for more optimal analysis. The mother wavelet function is denoted as $\Psi$ and is defined in Equation 2.1 [50],

$$
\Psi=\frac{1}{\sqrt{a}} \psi\left(\frac{t-b}{a}\right)
$$

where $a$ and $b$ represents the scale parameter and translational parameter of the wavelet, respectively. For a particular signal $x(t)$ and variations of the selected mother wavelet, the continuous wavelet transform (CWT) is given by Equation 2.2 [50].

$$
C_{x}(a, b)=\frac{1}{\sqrt{a}} \int_{-\infty}^{\infty} x(t) \psi^{*}\left(\frac{t-b}{a}\right) d t
$$

As shown in the CWT Equation 2.2, the mother wavelet acts as a window and the window property is dictated by the parameters $a$ and $b$. The scale parameter $a$ has a non-linear inverse relation to frequency and is different between mother wavelets. High scales (or low frequency) values correspond to the signal's global information, whereas the low scales (or high frequency) values would correspond to localized information [51]. With the scale parameter, it also preserves the signal's energy at each particular scale with the $\frac{1}{\sqrt{a}}$ coefficient. The translation parameter $b$ controls the translation of the mother wavelet window, in time. To fully visualize the wavelet transform of a signal, the coefficient generated for each sample and scale are used to form a matrix. By taking the energy of each wavelet coefficient, this will form an image known as a scalogram. With the visualization of the wavelet transform, it displays the local time-scale energy densities which is defined as $\left|C_{x}(a, b)\right|^{2}$. In regions where there are activity, such as large peaks or series of peaks, 

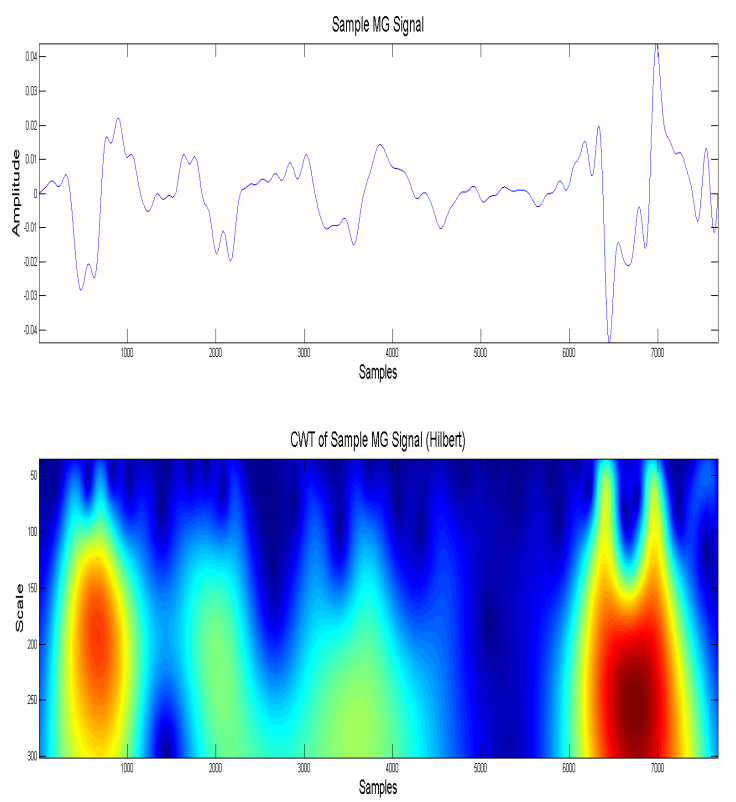

Figure 2.4: Example of a sample scalogram with continuous energy distribution.

will result in an energy distribution corresponding to where the peak occurred. The scale range when applying the CWT was limited to match the bandpass filter that was used. Although CWT is continuous for the computer implementation, the following discretized form was used in Equation $2.3[52]$.

$$
D_{x}(s, g)=\frac{1}{\sqrt{s}} \sum_{n=1}^{N} x(n) \psi^{*}\left(\frac{n-g}{s}\right)
$$

In Equation 2.3, the term $s$ represents the scale, $g$ represents the translation, and $D_{x}(s, g)$ represents the wavelet coefficients for the discrete time signal $x(n)$. An sample of the scalogram $\left(\left|D_{x}(s, g)\right|^{2}\right)$ that was formed from a filtered EOG signal is shown in Figure 2.4.

By selecting different mother wavelets, different characteristics of the signal could be emphasized. Each mother wavelet has their respective mathematical expression and could be altered to better suit the application. Some examples of commonly used wavelets are Mexican hat, Morlet, and Gaussian. In terms of the applications, the Mexican hat wavelets are frequently used in the field of geophysics for modeling seismic data, Morlet wavelets are widely used for detecting brief changes in the signal, and Gaussian wavelets are used to find single events that occur within a 
signal [50]. However, Morlet is also a common choice for biomedical applications such as cardiac related studies [53,54]. In this thesis, the mother wavelet will be selected based on their similarities with signal morphologies of interest. The decision and elimination process for mother wavelets will be discussed in Chapter 4 .

If the signal is only being observed in one domain, the other domain's resolution is compromised. From the time domain's perspective, it shows good temporal resolution, but lacks frequency resolution. In the case of frequency domain, it provides good frequency resolution of the signal, but suffers from time resolution. When discussing the notion of resolution for signal processing, the concept of Heisenberg's uncertainty principle needs to be understood. In essence, the uncertainty principle poses a limitation on the localization of time and frequency [55]. Approaches such as the short-time Fourier transform (STFT), lacks the necessary resolution to capture all the information due to its fixed window size. According to Equation 2.2, this approach overcomes this problem because of its adjustable window parameters in time and scale. Thus, with this varying window present in the wavelet transform, it yields better overall resolution in comparison to time and frequency domain only analysis, as well as STFT.

The wavelet transform has two distinct forms. The equation stated in Equation 2.2 is the continuous form. However, a discrete form of the wavelet transform also exists. Both forms are used in studies, but each form has their own unique purpose. The main difference between the continuous and discrete form is that the continuous has no issues when it comes to invariance properties. The discrete wavelet transform (DWT) is faster in comparison to its continuous counterpart because of its low computational time. However, the continuous form is more efficient and reliable as it preserves all the information without down-sampling [51]. For operations such as data compression, the DWT would be more suitable because it is able to reduce the size of the signal or information. If the main task was for analyzing or extracting features, the CWT approach would be more beneficial because all of the signal is conserved. By digitizing the CWT approach, the continuous aspect for the scale and translation parameter becomes discrete. However, the selection in scale increments or sample steps are adjustable and could be changed to suit the user's requirement. As the results of this thesis are dependent on the extraction of features, the CWT would be an appropriate 
choice.

For the application of this thesis, a continuous spectrum is required for feature extraction as the morphology of the signal is crucial. To achieve the continuous moments in the spectrum, there are two approaches: using complex wavelet functions or converting the signal into its analytical form. The analytical signal consists of a real and imaginary component and is computed through the Hilbert transform. The real component of the analytical signal is the original signal itself and the imaginary component is the $90^{\circ}$ shifted version of that signal. The algorithm itself makes use of the Fourier transform, but zero pads the negative portion and then applies the inverse transform on the zero padded signal. With the negative frequency components in the signal removed, spectral redundancy caused by the negative frequencies is eliminated [56]. All information from the realvalued signal is still preserved in the positive frequency components and results in continuous transition in the signal's spectrum. In Figure 2.4, it shows the scalogram of an analytical signal that has the Hilbert transform applied on the sample filtered EOG signal.

With the wavelet transform, different features could be extracted for various applications. Some of these well known wavelet features from existing features are entropy, energy, and ridges. Entropy or Shannon entropy provides a measure to the distribution of information. This wavelet entropy could measure the degree of chaos or disorder of the signal. Taking one step further, the relative wavelet entropy could be calculated by taking the entropy for different scale bands, which was demonstrated in a work done by Rosso et al. [57]. In the same work, the wavelet energy feature was expanded to a relative measure between different scale ranges and was referred to as relative wavelet energy. Both the relative wavelet entropy and energy makes use of sub-banding the whole scale range into smaller ranges. By sectioning these sub ranges, it could provide more localization to the structure of the signal. Another common wavelet feature is ridges. The ridge obtained from the wavelet transform is related to the signal's instantaneous frequency. A few examples of applying ridges are identifying non-linear systems [58], geophysics [59], and optical pattern analysis [60].

The use of wavelet transform has been explored throughout the decades for various applications. For biomedical applications, the wavelet transform provides various uses for different phys- 
iological signals. Some of these physiological analyses include observation of heart conditions through electrocardiogram (ECG) and phonocardiography, muscle activity through electromyography (EMG), and brain function through electroencephalography (EEG) [61,62]. Examples of other uses of wavelet transforms for biomedical applications are image compression, filtering, and image enhancement for medical imaging modalities such as magnetic resonance imaging (MRI), ultrasound, mammography, and many more [54].

The application of wavelet transform has been tried on EOG signals in attempt to extract eye movement features. The extraction procedure of two different eye movement features were observed and explained. One work focused on blink detection with the use of the DWT approach [63]. The wavelet approach made use of creating 4 frequency bands, which represent the majority of the signal's energy, and compared it with different thresholds. In another work [64], the DWT was applied to horizontal EOG signals formed by the difference between the right and left channel. The EOG signals were generated from only a PSG study in conjunction with EEG data. By decomposing the signal down into 9 levels with each level corresponding to a frequency range. The energy coefficients at levels 6 to 9 were extracted as features as those frequency ranges represented slow eye movements.

The wavelet transform is only a tool to help extract hidden information or features from the signal. A signal's embedded characteristics could be highlighted with the optimal selection of a mother wavelet and features. Although a mother wavelet can be selected due to a similar application, for its general intended purpose, or resemblance in waveforms, the performance or effectiveness of each wavelet can only be determined through some form of comparison. For this thesis, the use of pattern classification and linear discriminant analysis will quantify the usefulness of each type of mother wavelet along with their version of the extracted features, which is presented in chapter 4 . The intentions of pattern classification and its theory will be explained in the following section. 


\subsection{Pattern Classification}

The purpose of a pattern classification system is to distinguish between groups of data with the use of selected or common set of features. The primary objective of a pattern classifier is to be able to provide the most optimal segregation between different groups in an automated manner.

There is a variety of established pattern classification methods that have their own unique benefits. For this thesis however, the classification performance and accuracy were calculated through the use of Fisher's linear discriminant analysis. The following subsection will discuss a brief overview of linear discriminant analysis from "Pattern Classification" [65]. Additional information and more in depth explanations could be found in this book.

\subsubsection{Fisher's Linear Discriminant Analysis}

In pattern classification, the Fisher's linear discriminant analysis (LDA) [65] is a method that attempts to separate different data sets or groups using a linear expression. This is achieved with the use of data sets that are labeled by experts. With the provided label or class information, this method is known as a supervised learning. A supervised method is essential for medical applications because these labels are produced by clinicians and it will serve as a reference. These signals or data sets on their own have no meaning and does not help with discriminating which samples belong to patients with or without the disease. Pre-labeled samples will offer the designed classifier a reliable standard to compare to when discriminating the different groups as they are validated by medical experts themselves.

For the linear discriminant function, the objective of this method is to have a line orientated in way such that the samples are well-separated. This method's performance is dependent on how well the selected features segregates the different groups. Poorly chosen features will result in poor classification of the data sets and may conclude that the different groups are inseparable. Good performing LDA classifiers corresponds to how strong the selected feature is for discrimination or the simplicity of the separation task itself.

To form the LDA classifier, the minimum number of features required for discrimination is $c-1$, where $c$ represents the number of classes desired to be classified [65]. The Fisher's LDA 
function can be understood as projecting the data from $d$ dimensions onto a line. Samples with well-defined groupings could still exhibit a mixture of samples of different groups when projected onto an arbitrary line. The projection of the sample data set $x$ for the given class's weight vector $w$ with dimensions $d \times(c-1)$, the resultant projection $y$ is expressed as Equation 2.4 [65].

$$
y=\mathbf{w}^{\mathbf{t}} \mathbf{x}
$$

To obtain these weight vectors, the criterion function denoted as $\mathbf{J}(\mathbf{w})$ is given in Equation 2.5 [65]. This criterion function becomes maximum as the class separation is maximized. Within the criterion function, there are two distinct matrices, which indicate the scatter of the projected samples. The between-class scatter matrix $\mathbf{S}_{B}$ and within-class scatter matrix $\mathbf{S}_{W}$ is defined in Equation 2.6 and Equation 2.7 [65], respectively. The significance between the scatter matrix equations is that the between-class scatter represents the distribution between the classes themselves, whereas the within-class scatter represents the distribution of samples within the same class. These two equations are expressed in the general form for $c$ number of classes. In Equation 2.6, $n_{i}$ is the number of samples in class $i, \mathbf{m}$ is the total mean vector, and $\mathbf{m}_{i}$ is the sample mean in class $i$. For Equation 2.7, it could be expressed as the summation of scatter matrices $\mathbf{S}_{i}$ or the expanded form, which represents the summation of variance from each individual class.

$$
\begin{gathered}
\mathbf{J}(\mathbf{w})=\frac{\mathbf{w}^{\mathbf{t}} \mathbf{S}_{B} \mathbf{w}}{\mathbf{w}^{\mathbf{t}} \mathbf{S}_{W} \mathbf{w}} \\
\mathbf{S}_{B}=\sum_{i=1}^{c} n_{i}\left(\mathbf{m}_{i}-\mathbf{m}\right)\left(\mathbf{m}_{i}-\mathbf{m}\right)^{t} \\
\mathbf{S}_{W}=\sum_{i=1}^{c} \mathbf{S}_{i}=\sum_{i=1}^{c} \sum_{\mathbf{x} \in \mathbf{D}_{i}}\left(\mathbf{x}-\mathbf{m}_{i}\right)\left(\mathbf{x}-\mathbf{m}_{i}\right)^{t}
\end{gathered}
$$

By calculating the weight vectors, the classifier is trained with the given labeled samples. With the given training samples, a boundary is created that is optimized for this particular set of samples. The usefulness of the classifier is now dependent on the samples used for training. If the number of training samples are limited, it may not handle new data samples well if they vary significantly. For 
the classifier to be more robust or generalized, it is important to have sufficient amount of training samples. After understanding the theory of Fisher's LDA, the performance needs to be confirmed and applied. To eliminate the classifier's dependency on the training and testing samples, the use of cross validation evaluation method is needed.

\section{Cross Validation}

The concept of cross validation is a method to evaluate the model or classifier on how it responds to new data. There are many existing techniques to apply cross validation such as k-fold and leaveone-out method. For the k-fold method, the total number of samples is divided into $k$ equally sized sets of data. The leave-one-out method is based on the k-fold technique, but considers the extreme scenario of having the number of trials equal to the number of samples. This method is superior in comparison to the k-fold method since the classifier is trained on all the samples. However, this method could become computationally heavy if the total sample size is considerably large. Considering the small sample size of this thesis, the leave-one-out method is applicable.

The leave-one-out method chooses one sample in the data set to be a testing sample while the remainder of samples in the data set are used for training. After computing the LDA with these parameters, the test sample is returned to the data set and another sample is chosen to be used for testing. This process is repeated until all individual samples have been used as testing. In the theory of resampling, the leave-one-out mean is the sample average of the data when the $i^{\text {th }}$ point is removed as expressed in Equation 2.8 [65]. The next equation to be defined is the

jackknife estimate of the leave-one-out mean. The expression is given in Equation 2.9 [65]. Lastly, the jackknife estimate of the variance as expressed in Equation 2.10 [65]. The jackknife method is used because it extends the regular mean and variance equations to arbitrary statistics. By applying the leave-one-out method, $n$ number of results are generated and needs to be combined through the jackknife estimate. Expressing the variance from Equation 2.10 is advantageous because it is able to generalize to other estimators. The method to compute average could be altered from mean to median or mode. The jackknife resampling method generally provides a more adequate estimate of a statistic, but is computationally more complex. Nonetheless, the sample size used for this 
work is limited and is able to take advantage of the jackknife resampling technique.

$$
\begin{gathered}
\mu_{(i)}=\frac{1}{n-1} \sum_{j \neq i}^{n}=\frac{n \bar{x}-x_{i}}{n-1} \\
\mu_{(\cdot)}=\frac{1}{n} \sum_{i=1}^{n} \mu_{(i)} \\
\operatorname{Var}[\hat{\mu}]=\frac{n-1}{n} \sum_{i=1}^{n}\left(\mu_{(i)}-\mu_{(\cdot)}\right)^{2}
\end{gathered}
$$

\section{Classification Accuracy}

A classifier's accuracy could be statistically observed to quantify the classifier's performance that was derived from a set of features. The notion of accuracy in this scenario could be referred to the signal detection theory. There are four possible outcomes to consider: correctly detecting the signal when it is present, detecting a signal when it was not present, unable to detect the signal when present, and correctly detecting no signal when it is not present. To obtain the accuracy of the classifier itself, the expression is given in Equation $2.11[66,67]$.

$$
\text { Accuracy }=\frac{\text { Number of correct decisions }}{\text { Number of cases }}=\frac{T P+T N}{T P+F P+T N+F N}
$$

In Equation 2.11, the terms TP, FN, FP, and TN are equivalent to the outcomes presented by the signal detection theory. The term TP (True Positive) represents the number of cases of correctly detecting the signal when it was actually present, and FP (False Positive) represents the number of cases of detecting a signal when the signal was not present. In contrast, the term FN (False Negative) represents the number of cases of detecting no signal when the signal was present, and TN (True Negative) represents the number of cases where no signal was detected when the signal was actually not present. When observing the binary classifier in terms of disease and normal patients as the groupings, TP will represent correctly classifying disease patients as disease, and FP will represent classifying patients as disease but they were actually normal. The term TN would represent the correct classification of normal patients as normal, and FN represents the 
classification of patients as normal, but they actually have the disease. Two terms that needs to be taken into consideration when discussing accuracy are sensitivity and specificity. With the use of the four mentioned possible outcomes, the sensitivity and specificity of the classifier is determined by Equation 2.12 and Equation 2.13, respectively.

$$
\begin{aligned}
& \text { Sensitivity }=\frac{T P}{T P+F N} \\
& \text { Specificity }=\frac{T N}{T N+F P}
\end{aligned}
$$

By calculating the sensitivity and specificity, the overall classification could then be obtained. For comparisons between different features, this type of analysis would provide a means to quantify the usefulness of the feature. The ideal outcome of a classifier is to have a feature that could yield a high percentage for both sensitivity and specificity simultaneously. There are cases where one feature would exceed in one aspect but perform poorly in the other.

\section{Receiver Operating Characteristic}

The receiver operating characteristic or ROC curve is a graph that measures the change in sensitivity and 1-specificity of a binary classifier. A curve is formed by varying the decision boundary, which results in a change to both the sensitivity and specificity. Sensitivity is the horizontal axis while the 1-specificity is the vertical axis of the ROC curve. The purpose of this curve is to evaluate the performance of the test or the classifier and is insensitive to imbalanced data sets. A sample ROC curve is illustrated in Figure 2.5. In this figure, sensitivity is in the $\mathrm{Y}$ axis and specificity (plotted from 1 to 0 , which is 1-specificity) in the $\mathrm{X}$ axis. By observing the area beneath the curve, it correlates to the robustness of the feature used to discriminate the groups. Larger areas under the curve indicates more robustness in the feature that was selected to perform the classification. In the case where the curve is a linear line connecting the origin and the coordinate $(1,1)$, the classifier's performance would indicate that it is unable to distinguish between the groups. This evaluation tool is also useful for feature comparison for classifiers discriminating between two groups. The 


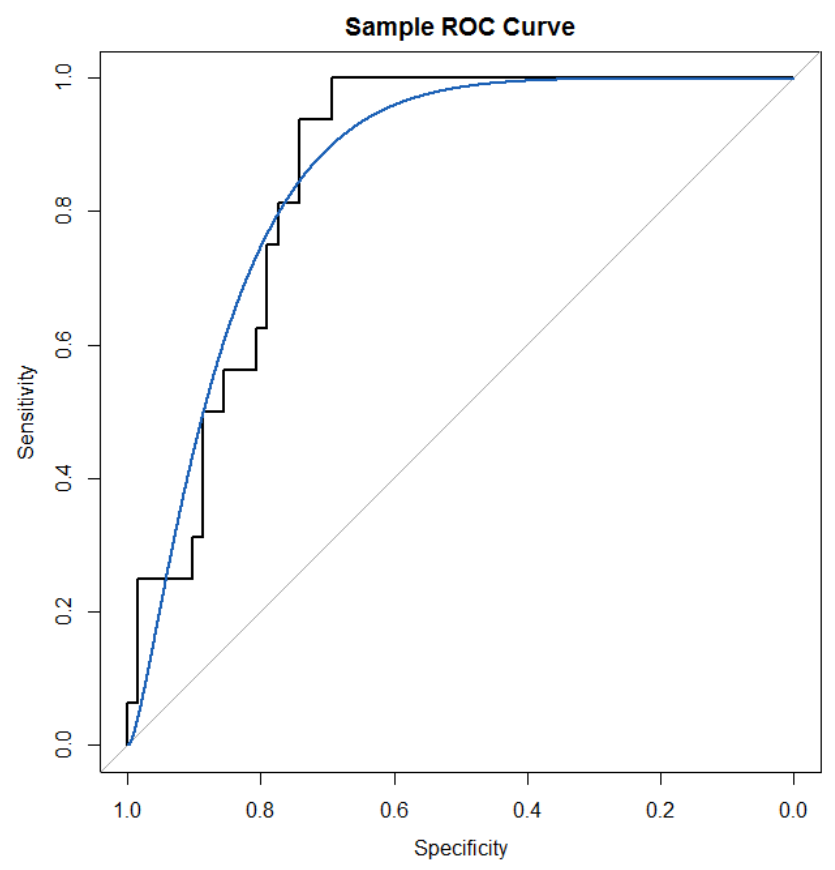

Figure 2.5: Sample ROC curve.

area under the respective feature's ROC curve would determine which feature is more sturdy and therefore, optimizes the selection of extracted features.

\subsection{Chapter Summary}

In this chapter, the background information for methodologies used for this work was presented. It introduced the mechanism of EOG generation, usage, and applications. The three different sleep tests were explained. Subsequently, relevant signal processing methods that were applied in this thesis, such as time domain analysis and wavelet analysis, were explained and justification of using a time-frequency or time-scale approach was provided. The existing works for the two mentioned techniques relating to EOG applications were briefly highlighted along with their approach. Lastly, the chapter concluded with the use of pattern classification and relevant classifier quantification using cross validation (leave-one-out), classification accuracy, and receiver operating characteristics. All mentioned techniques or methods were discussed as they are applied in the following chapters. 


\section{Chapter 3}

\section{Time Domain Analysis}

7 HIS chapter will present the time domain approach used for analyzing eye movements for

1 MG detection. The chapter will cover the pre-processing stages of the EOG signal, the process of feature extraction, feature selection, results, and discussion. Since time domain eye movement detection techniques are well known in sleep studies, it is rational to explore if timedomain features of EOG could detect MG. A block diagram displaying relevant parts of the thesis for this chapter is highlighted in Figure 3.1.

\subsection{Time Domain Eye Movement Feature Analysis}

As previously discussed in chapter 2, there are various existing works that have used different EOG signal based time domain eye movement features. Since MG affects ocular muscles, slow eye movements might better capture clues in detecting MG. Slow eye movements are mainly horizontal [64], and hence this thesis will focus on extracting features from horizontal eye movements. Similar to the work of [47], which uses slow eye movements, an initial study was conducted for MG detection and the results were published in [68]. This initial study demonstrated potential for using eye movement features for detecting MG. Further to the initial study, in this thesis we have expanded our time-domain analysis and optimized the features using a larger database. All methods and analysis were implemented using the MATLAB software [69] and the statistical analysis was performed using SPSS [70]. 


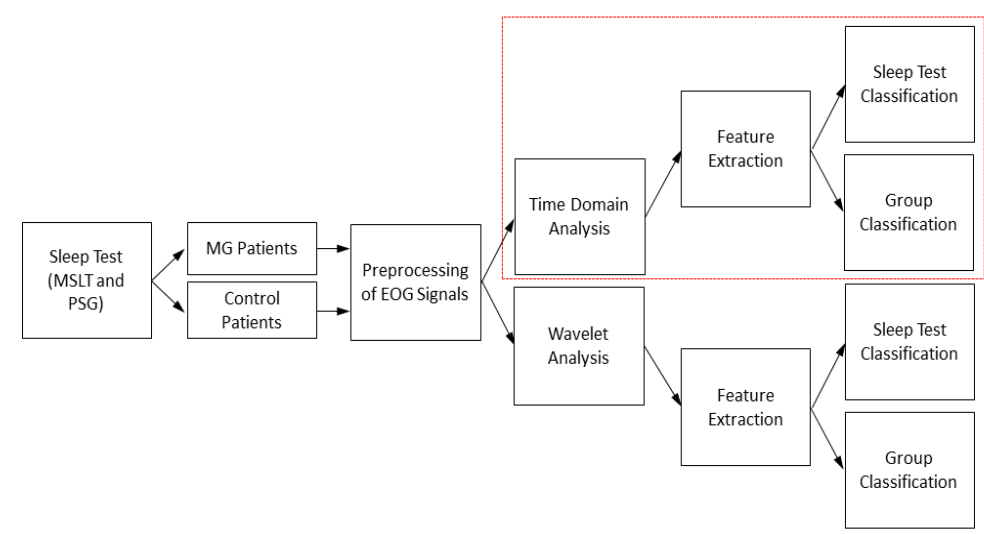

Figure 3.1: Block diagram showing the overview of chapter 3.

\subsubsection{Database}

The EOG signal database used in this thesis was obtained from Sunnybrook Health Sciences Centre. This study was approved by the Sunnybrook Research Ethics Board and written informed consent was obtained from the patients before participation. The databases consist of $8 \mathrm{MG}$ patients, who were diagnosed to have mild to moderate MG. Another 31 age- and gender-matched control patients served as a control for the analysis. All signals were sampled at $256 \mathrm{~Hz}$. Using the electrode configuration shown in Chapter 2, the EOG signals were recorded from the LOC, ROC, and VOC. In addition each of the patients had data collected with 3 pre-condition tests i.e. MWT, MSLT, and PSG. The protocol for taking these sleep tests occurs over the span of 2 days in order, starting with MWT during the day, PSG during the night, and MSLT the following day during daytime. The different tests capture contrasting traits of sleep neurophysiology, and may prove useful in detecting changes that occur in the ocular muscles due to MG. Of the 3 tests, MSLT and PSG are sleep related and these 2 test conditions had age- and gender-matched data, hence in this thesis we used only MSLT and PSG data for a fair comparison. Including both the MSLT and PSG conditions, we constructed 3 databases: 1. Group All - 62 control and 16 MG patients, 2. Group MSLT - 31 controls and 8 MG patients, and 3. Group PSG - 31 control and 8 MG patients. The 
rationale behind these 3 databases is that we were attempting to test if the proposed methods could detect MG with and without explicit matched pre-condition labels. Obviously a method that could detect MG irrespective of pre-condition test labels would be highly desirable. Group All is without pre-condition label, the other 2 are with MSLT and PSG labels. Although MSLT and PSG data are from the same patient, they were recorded at different time and with different pre-conditions. Eye movements are unique and time-varying hence, a MSLT and PSG signal recorded from the same patient at different times can be considered as an independent sample.

\subsubsection{Pre-processing}

Before feature extraction and any analysis could be performed, the given data had to be preprocessed into a more usable form. Figure 3.2 shows the different stages of the pre-processing procedures. The raw data for each patient was provided as one continuous signal containing information from all sleep stages together. With the use of the hypnogram data, the raw signal was divided into its corresponding sleep stage. By concatenating the segments of the raw signal labeled as the same stage, we form a signal representing all the events during that particular stage. The stages are categorized as Wake, Stage 1 to 3, and R, which signifies rapid eye movement (REM). Each sleep stage will vary from each other because different physiological activities occur in the

body and not all patients go through all stages of sleep. A person's sleep stage can be altered by previous sleep history, change in the person's environmental setting, medication, consumption of alcohol, and sleep disorders [71]. As for the purpose of this thesis, we used only the data from Wake stage as the other stages were not present for all the patients uniformly and hence performance comparison will be difficult. By focusing strictly on the Wake stage, the EOG signal consists of a combination of involuntary and voluntary eye movements since the subject is conscious. In comparison, the REM stage will be absent of voluntary eye movements from the subject as they are in deep sleep. Exploring the Wake stage alone could potentially determine if the effects of MG will cause changes in the number of movements as the subject's sleepiness increases.

Due to the nature of these sleep tests, each recorded EOG signal contains a vast amount of samples in time. These studies could vary between 5 to 7 hours. For computational efficiency and 


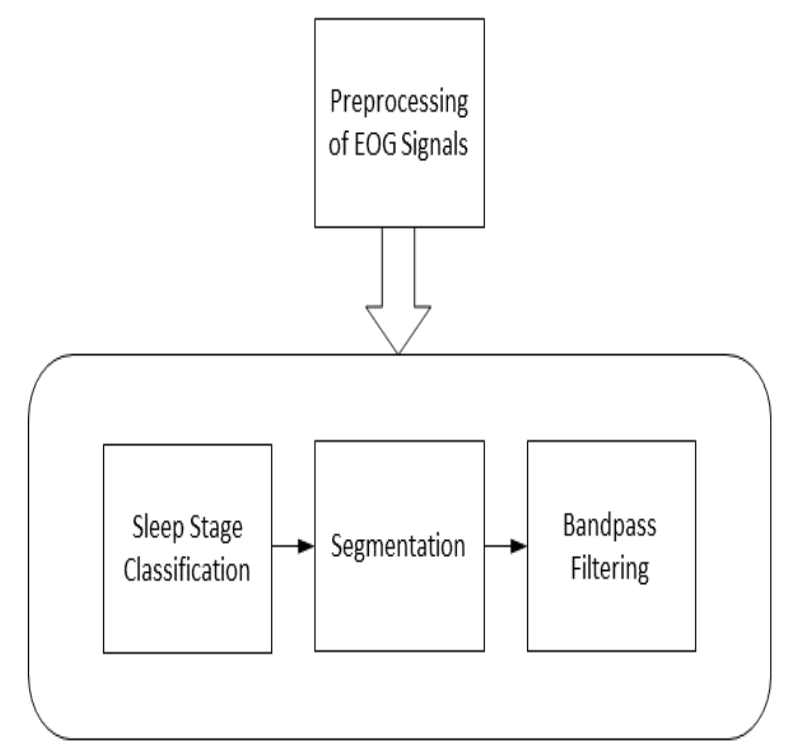

Figure 3.2: Block diagram showing the processes involved in the pre-processing procedure.

based on previous studies [47], only a portion of the raw signal was used for the analysis. The signal was limited to the first 10 minutes of each signal. These 10 minute time length EOG signals were then further divided into segments consisting of 30 seconds (based on hypnogram 30 second epochs) of samples for ease of computer processing. Considering the sampling frequency was 256 $\mathrm{Hz}$, each of the 20 segments contained 7680 time samples.

After obtaining a set of segmented signals from each EOG signal, the signals were filtered to remove any artifacts that may cause interference to the desired information. All the segmented signals were filtered using a bandpass filter limiting the frequency range of 0.2-1.75 Hz. From literature, different studies focused on different frequency ranges based on their desired eye movement feature. Some example frequency ranges for EOG applications could be found in [48,72-75]. However, the eye movement feature of interest for this thesis is slow eye movements. According to one study [64], it stated that the slow eye movement feature are mainly horizontal with a frequency range of 0.1-1 Hz. Although different variations of frequency cutoffs were experimented on the given data by trading off baseline artifacts vs retention of information, the final frequency range was selected to be $0.2-1.75 \mathrm{~Hz}$, which was similar to the suggested range. 


\subsubsection{Eye Movement Detection Algorithm}

The eye movement detection algorithm developed in this thesis consists of 3 main stages: normalization, peak detection, and pulse edge detection. Features were derived using the peak, time-topeak, and distribution of valid eye movement.

The first stage of the eye movement detection algorithm was the normalization stage. All segmented signals denoted as $\widetilde{x}[n]$ were amplitude normalized with respect to their segmented portion. Given the segmented signal $x[n]$, the normalization process is expressed in Equation 3.1. Mainly, this stage was used to scale the amplitude for all EOG signals to be in a similar range. Since each patient's EOG signal varies in their recorded amplitude, a fixed absolute threshold parameter cannot be used and hence amplitude normalization was used to set a percentage threshold based on peak amplitude. Care was also exercised through visual analysis that there were no outliers that can influence the amplitude normalization process.

$$
\widetilde{x}[n]=\frac{x[n]}{\max |x[n]|}
$$

The next stage of the eye movement detection algorithm was to detect the peaks that occur in the signal. The thresholds used in the algorithm were all relative in nature and in percentages to avoid absolute measures, which may vary among different patients and measurement conditions. The peak detection step of the algorithm can be seen in Equation 3.2.

$$
\left|\widetilde{x}\left[l_{i}\right]\right| \geq 0.25 \cdot \max |\widetilde{x}[n]|
$$

In Equation 3.2, the $l_{i}$ represents the index of the peak $p_{i}$ satisfying the condition. For peaks to be considered as a movement, certain conditions must be satisfied based on the expected characteristics of a normal eye movement. As the direction and movement of the eyes are normally parallel, there should be an anti-phase when comparing the left and right channel. Peaks that exhibit the anti-phase characteristic, these peaks are classified as genuine eye movements. Before this main criteria is applied, the detected peaks has to satisfy a peak threshold and pulse edge threshold. The threshold parameters used to make the decision were validated similar in a previous work [47]. 
Since the database used in the previous study was different, different peak thresholds were varied and was set to $25 \%$ for the given database. If the amplitude at the detected peak index exceeds this threshold, the peak index will be preserved and the next stage will be applied.

The last stage of the eye movement detection algorithm was validating the pulse edges. As peaks may satisfy the peak threshold, it may not be a proper movement if it does not return back to the baseline. These spikes could be caused by incomplete eye movements or fluctuations. To detect the pulse edges, samples before and after every peak index that passed the amplitude threshold stage was observed. The term rising and falling edge refers to the detected edge of the peak that comes immediately before and after the peak, respectively. For the $i^{\text {th }}$ peak that was detected in the segment, the rising edge must satisfy the condition of being the last time index that is less or equaling to $1 \%$ of the absolute maximum amplitude value of the segmented signal. Due to the initial normalization process, the absolute maximum value will always be 1 . The condition to obtain the pulse edges is shown in Equation 3.3.

$$
|\widetilde{x}[e(k)]| \leq 0.01 \cdot \max |\widetilde{x}[n]|, e= \begin{cases}1, \ldots, l_{i} & \text { if rising edge } \\ l_{i}, \ldots, M & \text { if falling edge }\end{cases}
$$

where $k$ corresponds to index of time samples of the segmented signal. The $e(k)$ is then sorted to identify the last/first found index that satisfies the condition depending on rising/falling edge. Then stored as a rising edge $R E_{i}$ or falling edge $F E_{i}$ corresponding to peak $p_{i}$.

As the range of time samples observed for the falling edge was from the index of the $i^{\text {th }}$ peak until the end of the sample, the time sample index for the detected peak was then added to the index of the detected falling edge to obtain the true index of the falling edge with respect to the segmented signal. The only exception to the rising and falling edge condition was if the detected peak was located at the beginning or the end of the segmented signal. For the scenario that the peak was detected at the first time index of the segmented signal, the rising edge would be defaulted as 1. If the peak was detected at the last time index, then the falling edge was defaulted as $M$. After satisfying the pulse edge detection stage, each movement should correspond to one peak. There are cases where multiple peaks are detected and uses the same pulse edges. Visually, these multiple peaks would appear within one large pulse. As there should only be one peak corresponding with 


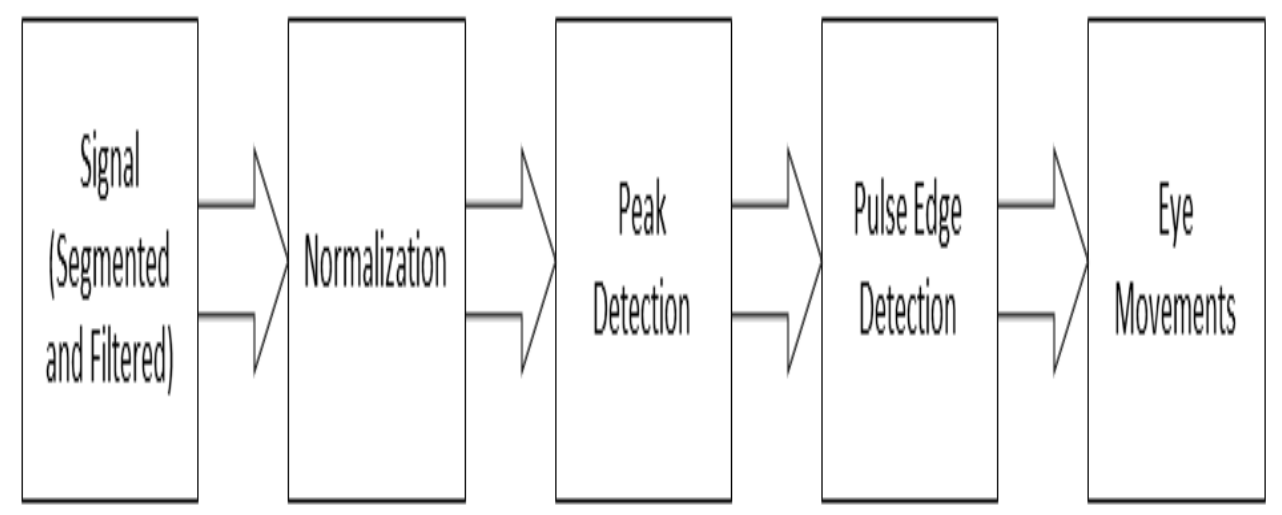

Figure 3.3: Block diagram displaying the process of the eye movement detection algorithm.

the pulse, the most dominant peak was taken to represent the group of peaks in the signal. The pulse edge stage of the algorithm will be iterated for $i$ peak locations detected.

Using the eye movement detection algorithm on the segmented signal, the resultant vector should contain only genuine movements. This algorithm only detects and confirms if the signal has eye movements and its authenticity. The block diagram representation of the eye movement detection algorithm is displayed in Figure 3.3. To visualize the process of the algorithm for a given sample EOG signal, Figure 3.4 shows the thresholds applied and the expected eye movement detected by the algorithm. The dashed red line represents the peak threshold, the dotted black line represents the pulse edge threshold, the open green circle represents the detected peak, and the solid green line represents the expected eye movement made by the patient.

After determining the detected peaks as genuine eye movements, we checked if the detected eye movement was in the horizontal direction. This requires the confirmation of peak amplitude and anti-phase between the left and right channel. This was achieved by determining if the right channel with the location of the detected $i^{\text {th }}$ peak from the left channel has satisfied two conditions. For simplicity, the right and left channel signal will be denoted as $\hat{x}_{R O C}[n]$ and $\hat{x}_{L O C}[n]$, 


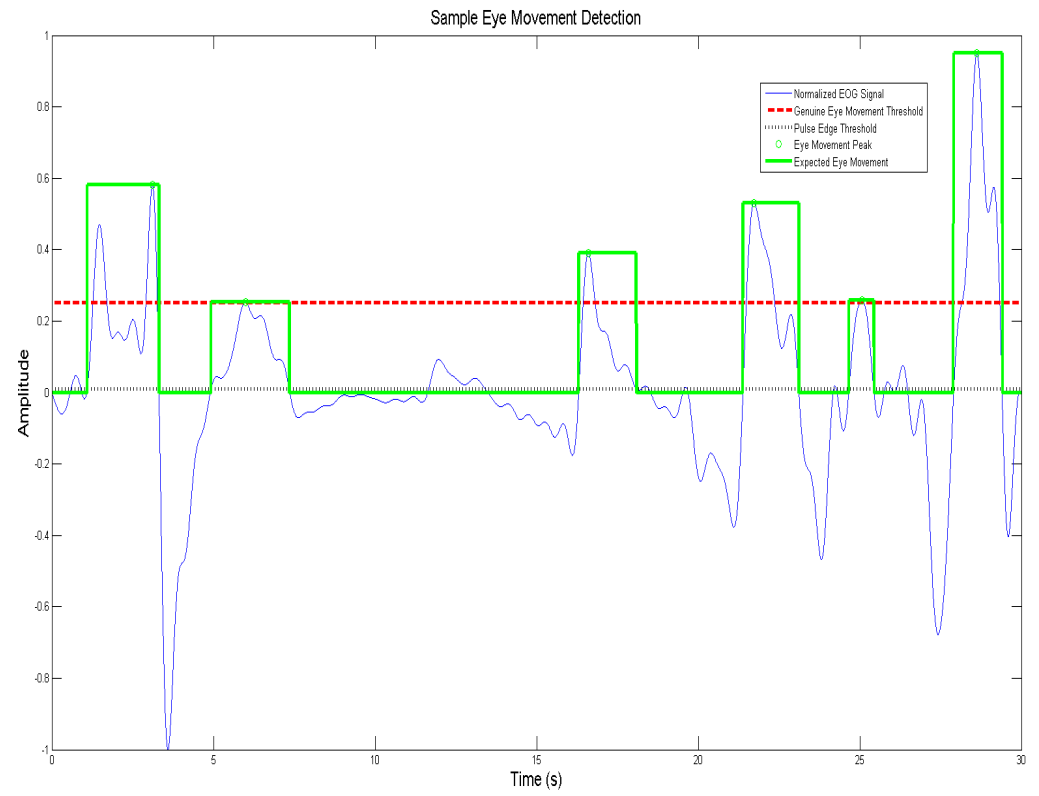

Figure 3.4: Illustration of eye movement detection algorithm using a sample EOG signal.

respectively. The first condition was to determine the anti-phase whether the sign of the $i^{\text {th }}$ peak location, from LOC, in $\hat{x}_{R O C}[n]$ was negative and $\hat{x}_{L O C}[n]$ was positive. In Figure 3.5, it illustrates the anti-phase verification process. For the same segment of the signal, the ROC is represented as a red, dashed line and the LOC is represented as a blue, dotted line. Secondly, the absolute amplitude of $\hat{x}_{R O C}[n]$ at $l_{L O C}$ from left channel was also valid by applying the same peak threshold value used earlier. Pulse edges that was paired with their respective detected peak were preserved accordingly. As the eye detection algorithm only detects the positive movement or the movement in the channel's direction, negative peaks were ignored. Thus, the anti-phase and signal amplitude conditions were also applied to the opposite case using the detected peak locations from the right channel. The overall horizontal, slow eye movement peaks and pulse edges from the right and left channel were combined and sorted in the order they occur. This algorithm is strict in the sense that it only approves of pure horizontal movements, which reduces ambiguity if the slow eye movements are also moving in an up or downward direction. In Figure 3.6, it shows the block diagram representation of the horizontal, slow eye movements algorithm. 


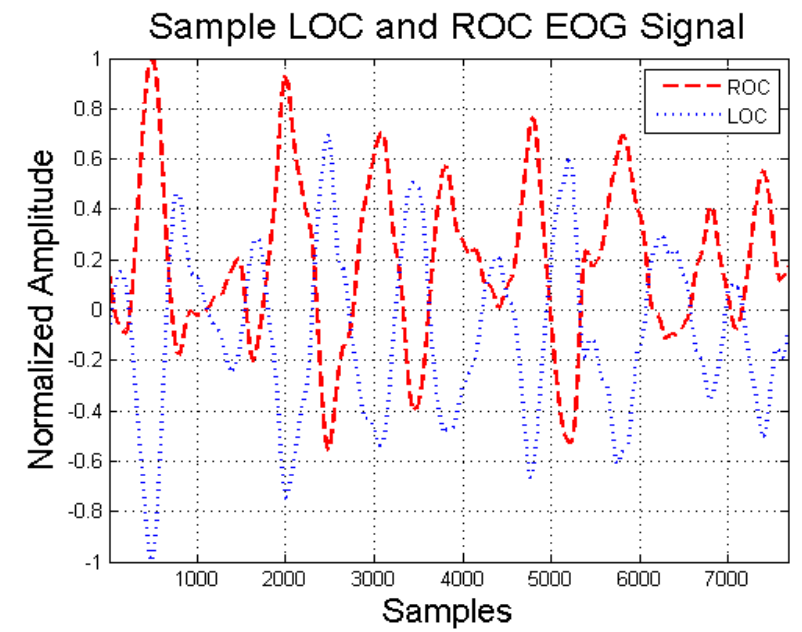

Figure 3.5: Sample LOC and ROC EOG signal illustrating anti-phase.

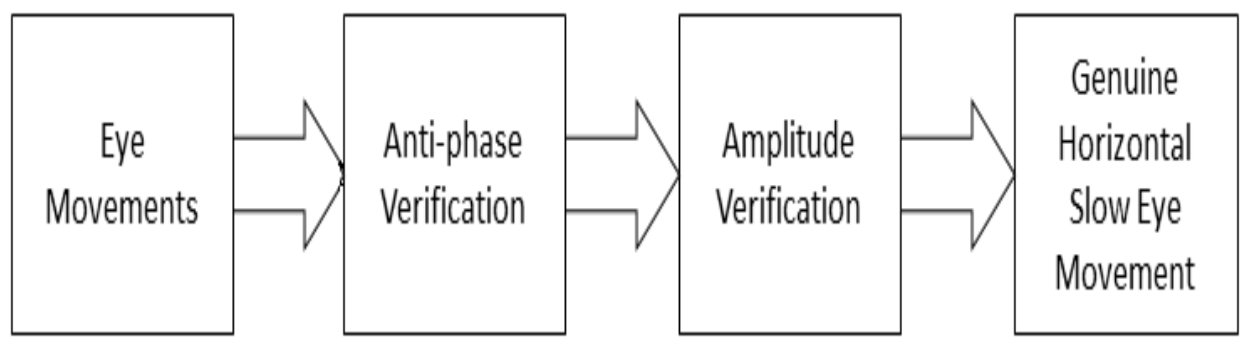

Figure 3.6: Algorithm for detecting genuine horizontal movements. 


\section{Algorithm Validation}

Only by satisfying the threshold values used in the peak and pulse edge detection stage, a peak could be considered as a valid eye movement. However, the results had to be clinically validated. The eye movement detection algorithm was validated by a neurologist from Sunnybrook Health Sciences Centre. Using $10 \%$ of randomly selected segments of data obtained from the segmentation stage, the detected movements were confirmed by the neurologist and was found to be reasonably accurate (84\% accuracy). A total count of the number of eye movements were compared with the neurologist's assessment. Each 30 second segment was labeled correct only if the neurologist considered the algorithm-detected movements were agreeable to their own assessment. The variance in the detected movements between the finalized eye detection algorithm used in this thesis and the initial version was the use of a bandpass filter. The difference between the initial was found to be $10 \%$.

\subsection{Feature Extraction}

In this section we present the feature extraction and selection process. Since MG affects ocular muscles, we expect the response of the eye movements of MG patients to be different compared to the control patients and hence we used this rationale in obtaining the following features.

\subsubsection{Average Horizontal Slow Eye Movement}

One of the features that was extracted for the analysis was the average horizontal, slow eye movements. This feature was of interest because SEM has correlation to sleep onset [64]. As this feature pertains to physiological activities about a person's condition, it may reveal useful information about the MG patients in comparison to the control patients. Using the eye movement detection algorithm and movement classification procedure explained in Section 3.1.3, number of horizontal SEMs were computed in each segment. The summation of the number of horizontal slow eye movements from each individual segment was then taken to obtain the total number of detected movements that has occurred in the 10 minute timespan. The average horizontal SEM counts is 
found using Equation 3.4, where $H_{k}$ represents the total number of horizontal SEM counts in a non-empty segment $k$, and $R$ represents the total number of non-empty segments. Empty segments were a result of no movements or peaks that are considered as a genuine movement by the eye movement detection algorithm. By removing segments without any valid detected movements, the number of horizontal, slow eye movements would show a better representation of the individual. Since the nature of MG could vary between patient to patient as well as moment to moment [1], an overall absolute count of movements may not be comparable between patients and segments. To overcome this, the average horizontal, slow movement per second was determined to acquire a more reasonable comparison between patients and groups.

$$
H_{\text {Average }}=\frac{\sum^{R} H_{k}}{30 \times R}
$$

\subsubsection{Average Rise and Fall Rate}

The other two features used for the analysis were rise and fall rate of the eye movements. These two features were extracted in a similar manner with the use of peak locations and pulse edges. This feature was used because it was expected that the MG disorder will influence the strength and/or speed of the eye movement. As clinically defined in the previous chapter, MG symptoms include the weakness and fatigue of muscles. If these symptoms are present in the ocular muscles, the strength and/or speed of the eye movement will be impacted. Rise rate represents the time it takes for a movement to reach its maximum or peak from zero. In contrast, the fall rate represents the time it takes for a movement to return to its original or resting position. As these features represent a rate of change, the rise and fall rate features can be considered as first order derivative with slopes in opposite directions. It was important to consider both amplitude and time duration of the eye movement. For each valid movement that was detected, the peak and its corresponding pulse edges are stored. The pulse edges come in a pair consisting of one rising edge and one falling

edge. By observing the $i^{\text {th }}$ peak location in the segment, the rise speed and fall rate is calculated by Equation 3.5 and 3.6, respectively. 


$$
\begin{aligned}
\text { Rise }_{\text {Rate }} & =\frac{p_{i}}{l_{i}-R E_{i}} \\
\text { Fall Rate }_{i} & =\frac{p_{i}}{F E_{i}-l_{i}}
\end{aligned}
$$

In both equations, the $p_{i}$ term represents the $i^{\text {th }}$ peak's normalized amplitude and $l_{i}$ term represents $i^{\text {th }}$ peak index of segmented signal. The $R E_{i}$ and $F E_{i}$ term represents the rising edge location and falling edge location, respectively. By applying Equation 3.5 and 3.6 to all segmented signals from one patient, the total number of rise and fall speeds elements were found. The average rise speed was calculated by taking the mean of the total number of rise speed elements over the total amount of time, in seconds. Similarly to the horizontal SEM feature, empty segments or segments that do not contain any movements by the algorithm could affect the overall count.

\subsection{Feature Selection}

We tested the discriminating ability of these features using two sample t-tests for control against MG and observed that the resulting $\mathrm{P}$ values were all statistically significant. We further used the stepwise feature selection process using SPSS [70]. By inputting all three features into SPSS using the stepwise method, the software determined which combination of features were best suited to separate the control and MG groups. Using the default $\mathrm{F}$ value to enter and to remove of 3.84 and 2.71 respectively [70], it was determined that the rise rate feature alone should be used as it displayed the highest $\mathrm{F}$ value for all three data sets.

\subsection{Results and Discussions}

In order to numerically quantify the ability of the features to classify MG from control subjects, an LDA based classifier was used with LOO cross validation. As explained in Chapter 2, LOO is an extreme form of cross validation, highly suitable for evaluating the classifier performance with small databases. The distribution of the features for the 3 databases (Group All, Group MSLT, and 
Table 3.1: LOO cross validated classification accuracy using Rise Rate for Group All.

\begin{tabular}{|c|c|c|c|c|}
\hline Method & Groups & MG & NC & Total \\
\hline \hline Cross-Validated & MG & $\mathbf{1 0}$ & 6 & 16 \\
\hline & NC & 8 & $\mathbf{5 4}$ & 62 \\
\hline$\%$ & MG & $\mathbf{6 8 . 7 5}$ & 31.3 & 100 \\
\hline & NC & 12.9 & $\mathbf{8 7 . 1}$ & 100 \\
\hline
\end{tabular}

Group PSG) are illustrated in Figure 3.7. A separate classification was performed using each of the databases and a stepwise feature selection was performed to choose the best feature.

\subsubsection{Group All}

All the 3 features (Average Horizontal SEM, Rise Rate, and Fall Rate) for 62 control and 16 MG patients were fed to the LDA classifier. A stepwise feature selection was performed, the analysis identified the Rise Rate feature to be the top performer with a F value and a P value of 28.689 and $8.754 \times 10^{-7}$, respectively. Using the Rise Rate feature, LOO based classification accuracies were computed and tabulated in Table 3.1. We obtained an overall classification accuracy of $83.3 \%$ with a sensitivity of $68.75 \%$ and a specificity of $87.1 \%$ ( $\mathrm{P}<<0.01$ i.e. statistically significant). Please note the overall classification accuracies is a weighted sum, which in this case favours the results as more weight is given for the $87.1 \%$ due to the larger control population. Although the sensitivity is much lower than the specificity, the overall accuracies is more biased towards the specificity. An ROC analysis was performed to evaluate the performance of the classifier for various thresholds. The area under curve (AUC) is a better metric for evaluation of the classifier performance especially for imbalanced data sets like the ones used in this study. An AUC can range between 0.5 and 1 . If the AUC approaches to 0.5 , it is interpreted as poor performance but if the AUC approaches 1, the performance is considered as excellent. Figure 3.8 shows the ROC curve with sensitivity in $\mathrm{Y}$ axis and specificity (plotted from 1 to 0 , which is 1 -specificity) in the $\mathrm{X}$ axis. All the ROC curves in this thesis follows the same convention. The AUC is also printed on the figure which is 0.88 . This demonstrates the robustness of the classification process and there by validates the results. 

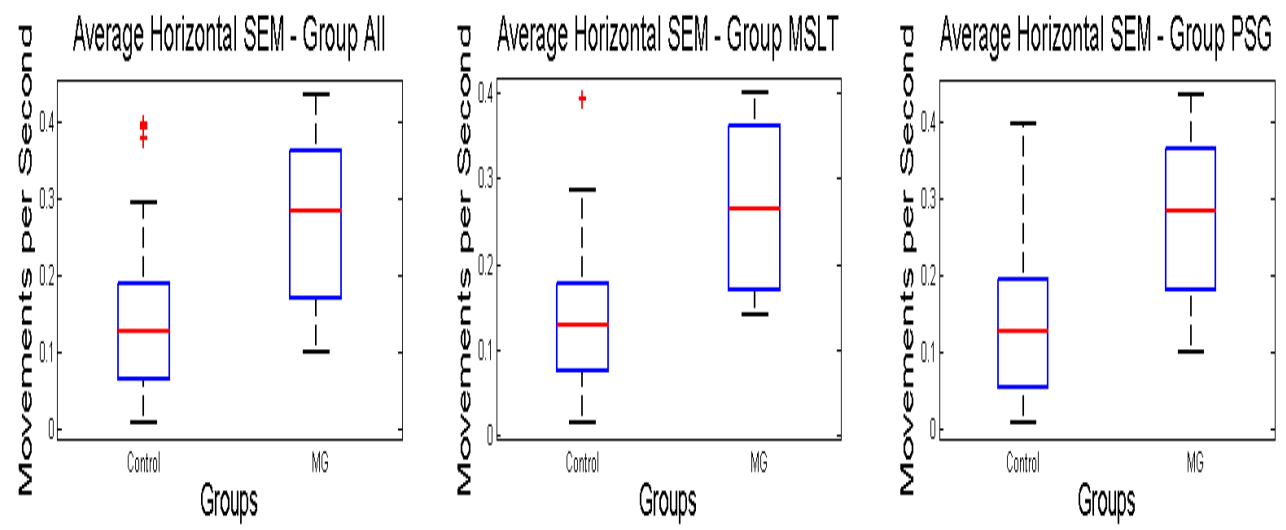

Average Horizontal SEM Rise Rate - Group All Average Horizontal SEM Rise Rate - Group MSLT Average, Horizontal SEll Rise Rate- Group PSG
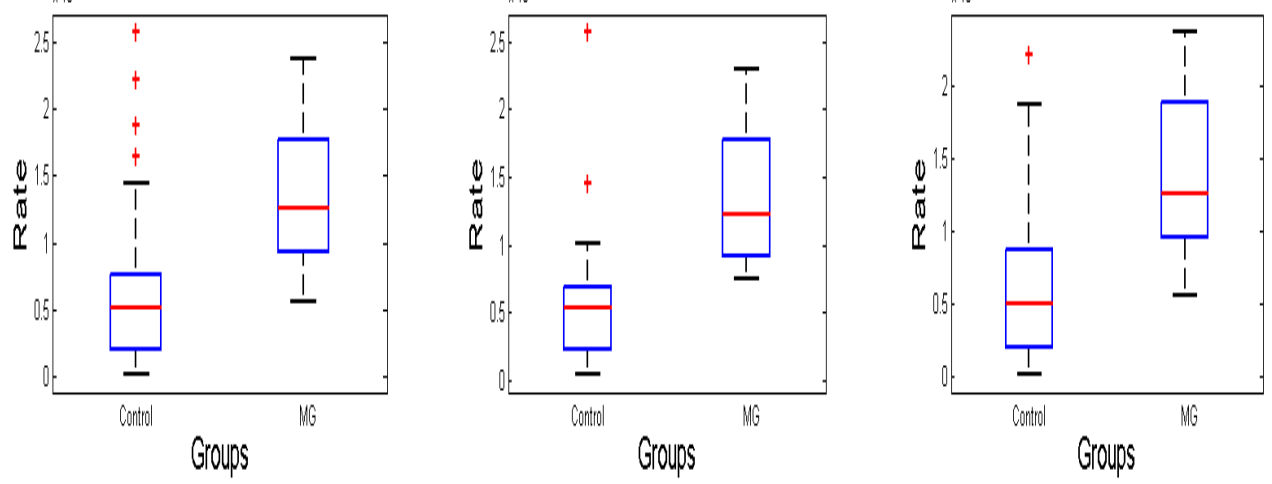

Average Hoizontal SEM Fall Rate - Group All
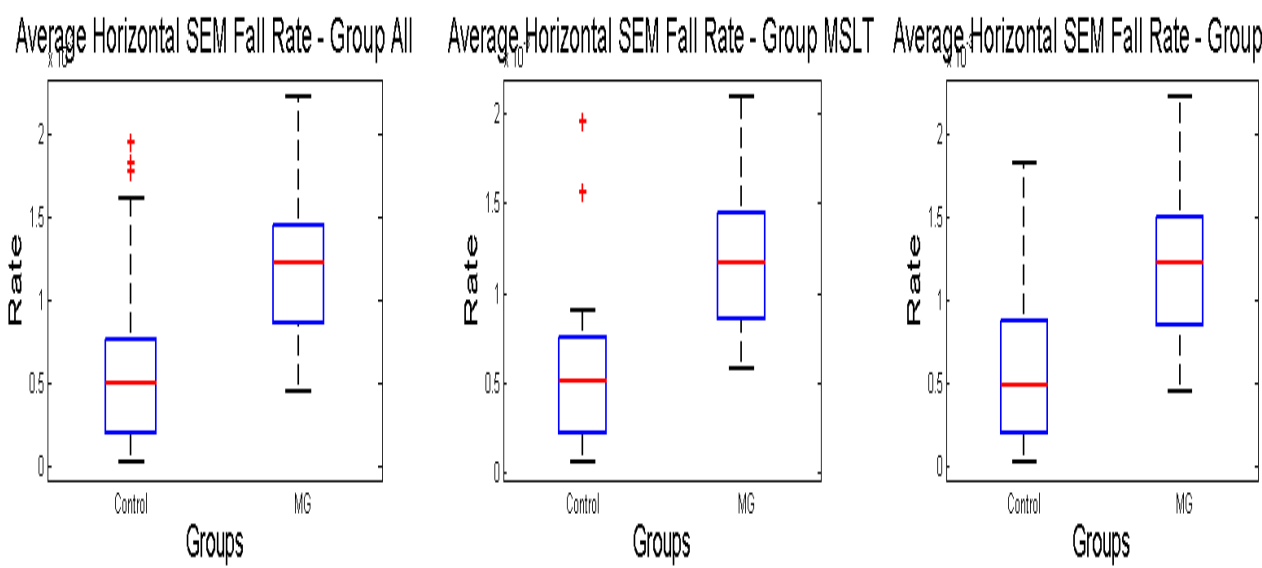

Figure 3.7: Boxplot figures for each group and feature. Each column represents a group while each row represents a feature. 
Table 3.2: LOO cross validated classification accuracy using Rise Rate for Group MSLT.

\begin{tabular}{|c|c|c|c|c|}
\hline Method & Groups & MG & NC & Total \\
\hline \hline Cross-Validated & MG & $\mathbf{5}$ & 3 & 8 \\
\hline & NC & 4 & $\mathbf{2 7}$ & 31 \\
\hline$\%$ & MG & $\mathbf{6 2 . 5}$ & 37.5 & 100 \\
\hline & NC & 12.9 & $\mathbf{8 7 . 1}$ & 100 \\
\hline
\end{tabular}

\subsubsection{Group MSLT}

Similar to the previous section, a stepwise feature selection was performed, the analysis identified the Rise Rate feature to be the top performer with a F value and a P value of 15.920 and 0.0003 . Using the Rise Rate feature, LOO based classification accuracies were computed for Group MSLT (31 control and $8 \mathrm{MG}$ ) and tabulated in Table 3.2. We obtained an overall classification accuracy of $82.1 \%$ with a sensitivity of $62.5 \%$ and a specificity of $87.1 \%(\mathrm{P}<<0.01$ i.e. statistically significant). Figure 3.9 shows the ROC curve with sensitivity in $\mathrm{Y}$ axis and specificity in the $\mathrm{X}$ axis plotted for various thresholds. The AUC is also printed on the figure which is 0.91 .

\subsubsection{Group PSG}

The stepwise feature selection analysis identified yet again the rise rate feature to be the top performer with a $F$ value and a $P$ value of 12.457 and 0.001133 . Using the rise rate feature, LOO based classification accuracies were computed for Group PSG (31 control and $8 \mathrm{MG}$ ) and tabulated in Table 3.3. We obtained an overall classification accuracy of $82.1 \%$ with a sensitivity of $62.5 \%$ and a specificity of $87.1 \%$ ( $\mathrm{P}<<0.01$ i.e. statistically significant). Figure 3.10 shows the ROC curve with sensitivity in $\mathrm{Y}$ axis and specificity in the $\mathrm{X}$ axis plotted for various thresholds. The AUC is also printed on the figure which is 0.87 .

Although the stepwise feature selection process identified the rise rate as the best feature for all the 3 data sets, we did perform the classification with other features and found them to have similar accuracies. The overall accuracies and $\mathrm{P}$ values were ranging between $74.4 \%$ to $84.6 \%$ and $4.657 \times 10^{-3}$ to $4.0 \times 10^{-6}$. We also tested the combination of these features and none of 


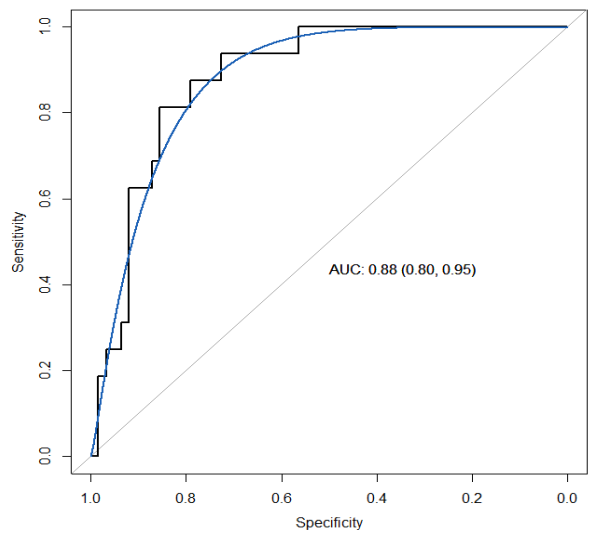

Figure 3.8: ROC curve for classifier using rise rate for Group All.

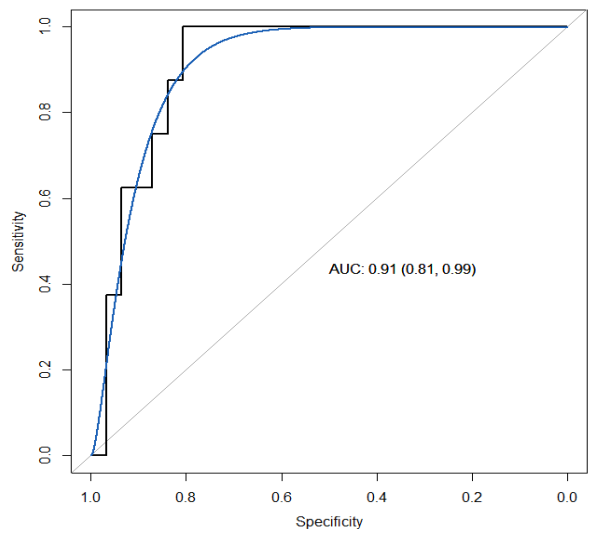

Figure 3.9: ROC curve for classifier using rise rate for Group MSLT.

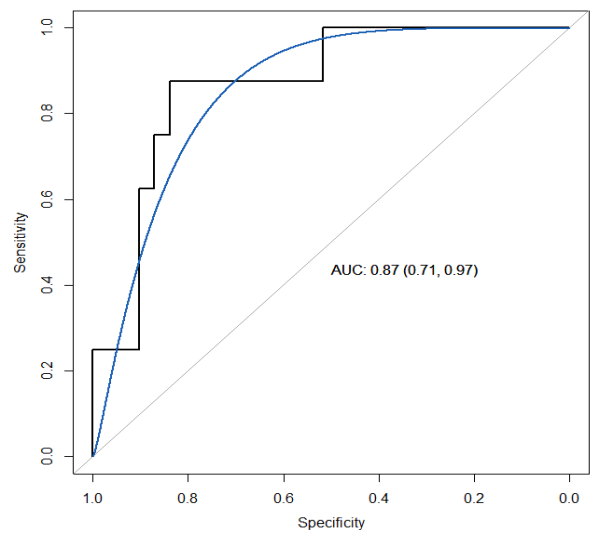

Figure 3.10: ROC curve for classifier using rise rate for Group PSG. 
Table 3.3: LOO cross validated classification accuracy using Rise Rate for Group PSG.

\begin{tabular}{|c|c|c|c|c|}
\hline Method & Groups & MG & NC & Total \\
\hline \hline Cross-Validated & MG & $\mathbf{5}$ & 3 & 8 \\
\hline & NC & 5 & $\mathbf{2 6}$ & 31 \\
\hline$\%$ & MG & $\mathbf{6 2 . 5}$ & 37.5 & 100 \\
\hline & NC & 16.1 & $\mathbf{8 3 . 9}$ & 100 \\
\hline
\end{tabular}

the combinations produced better results than rise rate alone. Even if the overall classification was determined to be $84.6 \%$, it was as significant in comparison the rise rate feature.

Based on the above presented results, it is evident that a single time-domain feature (Rise Rate) could successfully classify MG from control data with or without labels. The ability to classify MG even without matched pre-condition labels indicate that the EOG signals inherently capture some of the MG clues. Observing the boxplots of the distribution of the rise rate, it makes sense that the rise rate of $\mathrm{MG}$ patients were higher in $\mathrm{MG}$ than the control group. A possible explanation for the obtained results was that more involuntary/voluntary eye movements were presented in MG. Probably these eye movements were arising from MG attempting to control their eye movement against the muscle fatigue. These eye movements potentially exhibiting muscular twitching, eye movements could reach its maximum quicker in comparison. Comparing the control and MG patients in the average SEM boxplots, it appears that MG patients achieved more eye movements during the 10 minute time period. In order to have more eye movements per unit time, the rate at which the movements are invoked has to be higher.

All 3 classification exercises resulted in an overall accuracy of $80 \%$ which illustrates the potential of using EOG signals for the application. However the sensitivity of the proposed approach is much lower than the specificity (i.e. the technique is able to detect control as control is better than $\mathrm{MG}$ as $\mathrm{MG}$ ). If early detection of $\mathrm{MG}$ is our end goal, than a balanced high sensitivity and specificity would be more desirable. In the above presented results, due to the weighted mean approach for computing the overall accuracy this imbalance in sensitivity and specificity is masked. Hence, an ROC analysis was also performed to validate the discriminating ability of the proposed method with imbalanced data sets. 
The obtained results are heavily dependent on the normalization and segmentation process. As normalization is a common procedure, the amplitude normalization used in this work is dependent on whether sudden or random spikes exists in the signal that was not generated by the subject. However, by using the segmentation, it reduces the effect of these sudden large pulses from the signal by limiting it to a certain segment. By fixing the time length of the segment, some eye movement counts could be affected as the counts may be viewed as two independent movements depending on where the segment ends. Taking larger time lengths for segmenting will decrease the likelihood of repeated or misinterpreted movements but it will increase the effects of potential irregular spikes. Thus, there is a tradeoff for how large or small the selected segmented window size is.

While the time-domain features performed well in the classification exercise, the sensitivity of the technique is poor and moreover it requires many manual interventions, iterative thresholds in processing the data. If the information content is all hidden in the eye movement pulses or morphology of the EOG signals, which is time-varying, we should be able to automate this process using advanced signal processing tools. Since the eye movements are represented by scaled

versions of pulses (both in time and amplitude), we should be able to capture these adaptively by wavelet transform unlike the time-domain methods. Wavelet transform also provides a variety of basis functions that could capture different characteristics of these eye movement pulses.

\subsection{Chapter Summary}

In this chapter, we presented the time-domain approach for detecting MG. The chapter presents details on different database subsets, pre-processing, feature extraction, and classification results. The three features used for the analysis were horizontal slow eye movements, rise rate, and fall rate. The proposed method performed well with over $80 \%$ overall classification accuracies with or without pre-condition labels. The sensitivity of the technique was lower than the specificity. The results were validated both by LOO (small databases) and ROC (imbalance data sets). Considering the success of the rise rate, in search of a morphological feature that reflects this characteristic, this thesis will further explore wavelet transform in the next chapter. 


\section{Chapter 4}

\section{Wavelet Domain Analysis}

F

ROM the previous chapter, it is evident that EOG based analysis for MG detection is viable with satisfactory performance and accuracy. The time-domain algorithm presented in the previous chapter involves experimental thresholds and manual interventions in order to extract discriminative features that showed separation between the control and MG groups. To overcome these difficulties, an adaptive signal processing technique would be ideal. Since most of the information is contained in the eye movement pulses (which are scaled versions of self similar EOG signal morphologies), wavelet analysis might offer a better solution for the application. Hence in this chapter we explore the application of the wavelet analysis for MG detection. The chapter begins with motivation of using wavelet analysis and choice of wavelet functions, then discusses the process of feature extraction and classification using the 3 data sets (Group All, Group MSLT, and Group PSG) defined in the previous chapter. Finally, this chapter will conclude with a summary of the highlights that were presented. The portion of the thesis block diagram in Figure 4.1 relevant for this chapter is highlighted.

\subsection{Wavelet Analysis}

The CWT as explained in Chapter 2 was applied to the pre-processed EOG signals. The preprocessing was identical to the time domain approach explained in previous chapter. Before we could perform the wavelet analysis, we need to select appropriate wavelet basis function for the application in hand. 


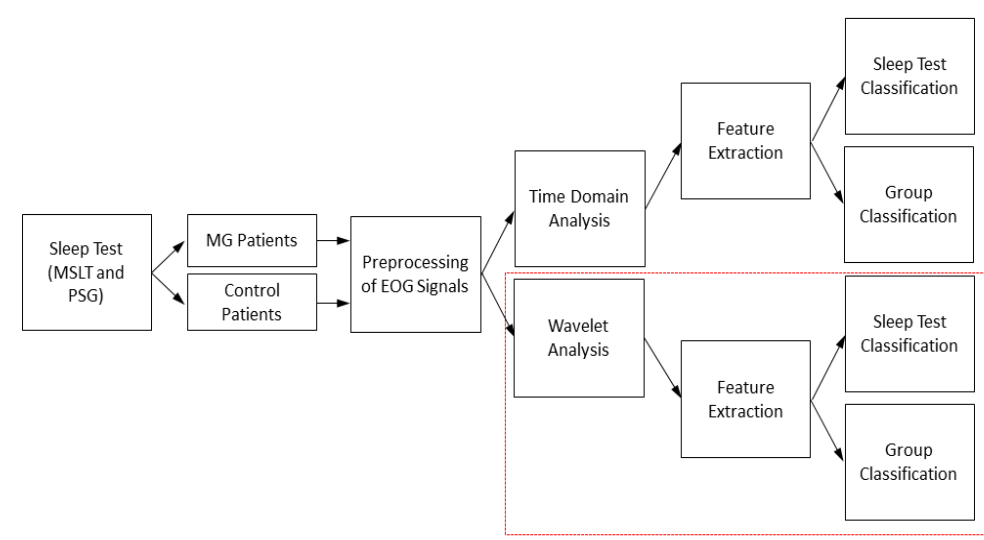

Figure 4.1: Block diagram showing the overview of chapter 4.

\subsubsection{Wavelet Selection}

To apply the wavelet transform, a mother wavelet needs to be selected. As there are various choices of wavelets, each individual wavelet provides certain properties as described in Chapter 2. The purpose of these properties were to emphasize different characteristics from the signal. Our motivation of using wavelet analysis was that EOG morphologies resemble scaled versions of an ideal eyemovement pulses, the choice of wavelet functions were constrained by their structural similarity to the eye-movement pulses. This narrowed down our choices to 3 wavelet functions: Gaussian, Mexican Hat, and Morlet. Their similarity with the eye-movement pulses are shown using a random EOG segments for MG and control patients in Figures 4.2 . In the figure, the scale was chosen to approximately match the width of the average peak in the sample signal. The details of these wavelets are provided below.

\section{Gaussian Wavelet}

The Gaussian wavelet function is expressed in Equation 4.1 [69], where the term $m$ represents the derivative order. Depending on the order of the Gaussian function, the symmetry and number of peaks will vary. If $m$ is odd, the function will be symmetrical about the origin and if $m$ is even, the 

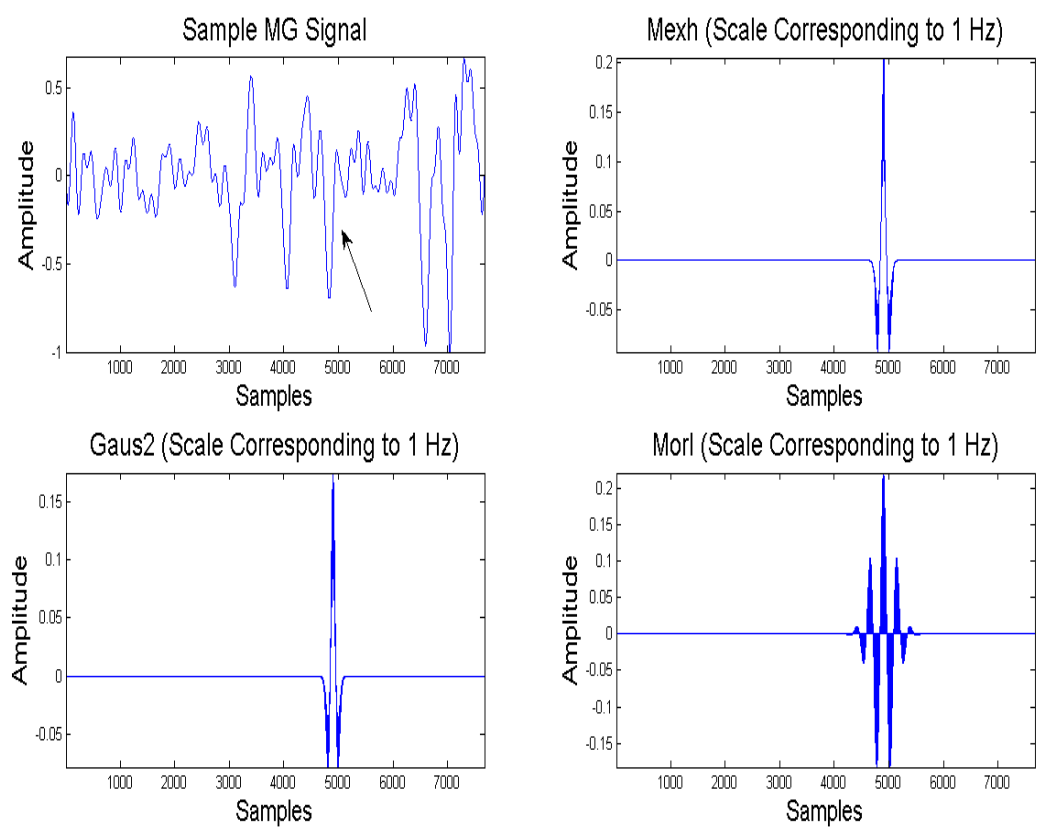

Figure 4.2: Sample MG EOG morphology compared to different wavelet structures.

function is symmetrical at the vertical axis.

$$
\operatorname{gaus}(n, m)=C_{m} \frac{d^{m} \exp \left(-n^{2}\right)}{d n^{m}}
$$

\section{Mexican Hat Wavelet}

The expression for the real Mexican Hat wavelet function is shown in Equation 4.2 [69]. The Mexican Hat is similar to the second order Gaussian wavelet function.

$$
\operatorname{mexh}(n)=\frac{2}{\pi^{\frac{1}{4}} \sqrt{3}} \cdot \exp \left(\frac{-n^{2}}{2}\right) \cdot\left(1-n^{2}\right)
$$

\section{Morlet Wavelet}

The expression for the real Morlet wavelet is given in Equation 4.3 [69]. The Morlet wavelet is based on a Gaussian function but contains a modulating sinusoidal term. 


$$
\operatorname{morl}(n)=\exp \left(\frac{-n^{2}}{2}\right) \cdot \cos 5 n
$$

Having chosen the mother wavelet functions for analysis, we now want to target specific signal morphologies that would differentiate MG from control group. Our visual inspection of the data indicated the MG patients had narrower eye movements (or movements with higher rise rate). Although signals from the control patients also displayed narrower movements, these were much more frequent for MG. In addition, we were encouraged by our results of the time-domain technique where MG patients exhibited higher rise rate. Hence, we focused on these narrower eye movements with high rise rates. To target these narrower peaks, we need to choose appropriate scales that are wavelet dependent.

\subsubsection{Scale Selection}

As mentioned in Chapter 2, the scale term in the wavelet transform will vary according to the mother wavelet that is selected. In order to make appropriate comparisons between each wavelet for the same feature, the frequency range has to be the same. Scale and frequency are inversely related, but not linear. Thus, the conversion process for a particular scale will produce a pseudofrequency. The conversion process from scale to its corresponding pseudo-frequency $F_{a}$ is calculated with Equation 4.4 [69].

$$
F_{a}=\frac{F_{c}}{a \cdot \Delta}
$$

In Equation 4.4, the $F_{c}$ term represents the central frequency of the selected wavelet in $\mathrm{Hz}, a$ represents the desired scale to be converted, and $\Delta$ represents the sampling period. The selected frequency range for all wavelet functions was chosen to be from $0.2 \mathrm{~Hz}$ to $1.75 \mathrm{~Hz}$ (which is the bandpass cutoff of our pre-processing). By varying a range of scale values within this range with Equation 4.4, the corresponding pseudo-frequencies can be found. As the exact scale band that pertains to the information that uniquely distinguishes MG is unknown, in the feature extraction process optimal scale bands were searched. However, we focussed on the lower scale ranges 
(higher frequency bands) as we were looking for the narrower eye movements which also correlates with the rise rate feature in the time-domain approach.

\subsection{Feature Extraction}

There are numerous wavelet features that exist in literature, but different features will produce different results. For this thesis, scale band energy and scale distribution width were attempted. As both of these features relate to scale band characteristics, it may be associated to specific signal morphologies. If MG affects the eyes non-uniformly, only one of the channels may capture the MG signal morphology. By treating each channel as a separate source, we obtain a feature per channel.

\subsubsection{Scale Band Energy}

For extracting meaningful selective scale band energy features that are comparable, all signals were energy normalized before CWT was performed. This was done to eliminate influence of energy discrepancies in the signal to affect the results. In other words, all signals will have unit energy to start and their relative scale band energies are then comparable in the time-scale domain. The normalization in energy is expressed in Equation 4.5, given $x[n]$ was the segment signal.

$$
x_{\text {norm }}[n]=\frac{x[n]}{\|x[n]\|}
$$

After the energy normalization, comparison between the identical subbands in the time-scale domain is possible. As mentioned in Section 4.1.2, we target specific localized scale bands that might contain the morphology of interest in detecting MG. The energy captured in a scale band is given by Equation 4.6.

$$
S B E_{x}=\sum_{g=1}^{l} \sum_{s=q_{1}}^{q_{2}}\left|D_{x}(s, g)\right|^{2}
$$

In Equation 4.6, the $D_{x}(s, g)$ term is the wavelet coefficient at time sample or translation $g$, scale $s$, the $l$ term represents the total number of columns or time samples, and the $q_{1}: q_{2}$ represents 
the scale band. As explained earlier, we focused on scales that were corresponding to the higher frequencies and found the peak performance observed for the given database using Mexican Hat wavelet for a frequency range of 0.8 to $1.42 \mathrm{~Hz}$. After obtaining the total scale band energy value for each segment, a median based averaging method was used. Since the wavelet approach is adaptive and unlike time domain features, we do not pre-select locations (i.e. eye movements) for analysis. However, there may be instances where a particular segment may have abnormally low or high feature values. Hence, a median approach is preferred, which is more resilience to the influence of potential outliers from the data segments. By separating the LOC and ROC channels, the SBE feature extracted from both channels could be considered as a separate feature. To see the effects of both channels as a whole, the SBE feature from LOC and ROC was averaged together to form a mean SBE feature.

\subsubsection{Scale Distribution Width}

The other feature that was explored in this thesis was the scale distribution width (SDW) [76]. The SDW is a wavelet feature, which captures the scale dependent energy profile of the scalogram. This feature was chosen in addition to scale band energy because if a certain scale band was more dominant in comparison to the rest, it could imply that the information is heavily localized in that particular scale region and how wide it distributes itself across the scale range. To calculate the SDW, the energy in each scale was computed and repeated for all the scales that were used and expressed in Equation 4.7. The $s$ term represents the scales and $g$ represents the range of translations. The full scale range of $s$ is extended until a Gaussian profile could be achieved.

$$
P_{\text {scale }}[s]=\sum_{g}\left|D_{x}(s, g)\right|^{2}
$$

To obtain the actual width from the scale distribution profile, the half maximum value of the

scale energy profile was found and used as a threshold to calculate the scale width. This width corresponds to a set of scales that are dominant in representing the signal characteristics. 


\subsection{Feature Selection}

Similarly from Chapter 3, the discriminating ability of the extracted features were tested using two sample t-tests for control against MG. It was observed that the resulting $\mathrm{P}$ values were only statistically significant for the SBE feature for all group classifications and all wavelet functions. As the SBE feature from LOC, ROC, and the mean showed significance in their respective $\mathrm{P}$ values, a stepwise feature selection was required to select the best feature that was statistically most suited for the separation of MG and control. Of the three selected wavelet functions, Mexican Hat performed relatively the best among the wavelet functions and hence, the detailed results are only presented for the Mexican Hat wavelet function in the following section. The SDW feature performed inconsistently with poor classification results and insignificant $\mathrm{P}$ values hence, it was not considered for further analysis.

\subsection{Results and Discussion}

By using an LDA based classifier, the ability of the features to separate MG and control was numerically quantified with LOO for cross validation. As the database size for the wavelet analysis is small, the LOO cross validation method was still applicable and effective to evaluate the classifier's performance. The distribution of the features for the 3 databases (Group All, Group MSLT, and Group PSG) using the Mexican Hat wavelet function are illustrated in Figure 4.3. Each row corresponds to the channel used to extract the EOG signals from. From top to bottom, it represents the channels from LOC, ROC, and the combination of LOC and ROC. Separate classifications were performed by using each individual database and a stepwise feature selection was performed to select the most suited extracted feature.

\subsubsection{Group All}

Although other features were attempted, the three features that stood out above the rest were the SBE features from LOC, ROC, and the combination of the two. The classification performance of each feature for the 62 control and 16 MG patients were used for the LDA classifier. After applying 

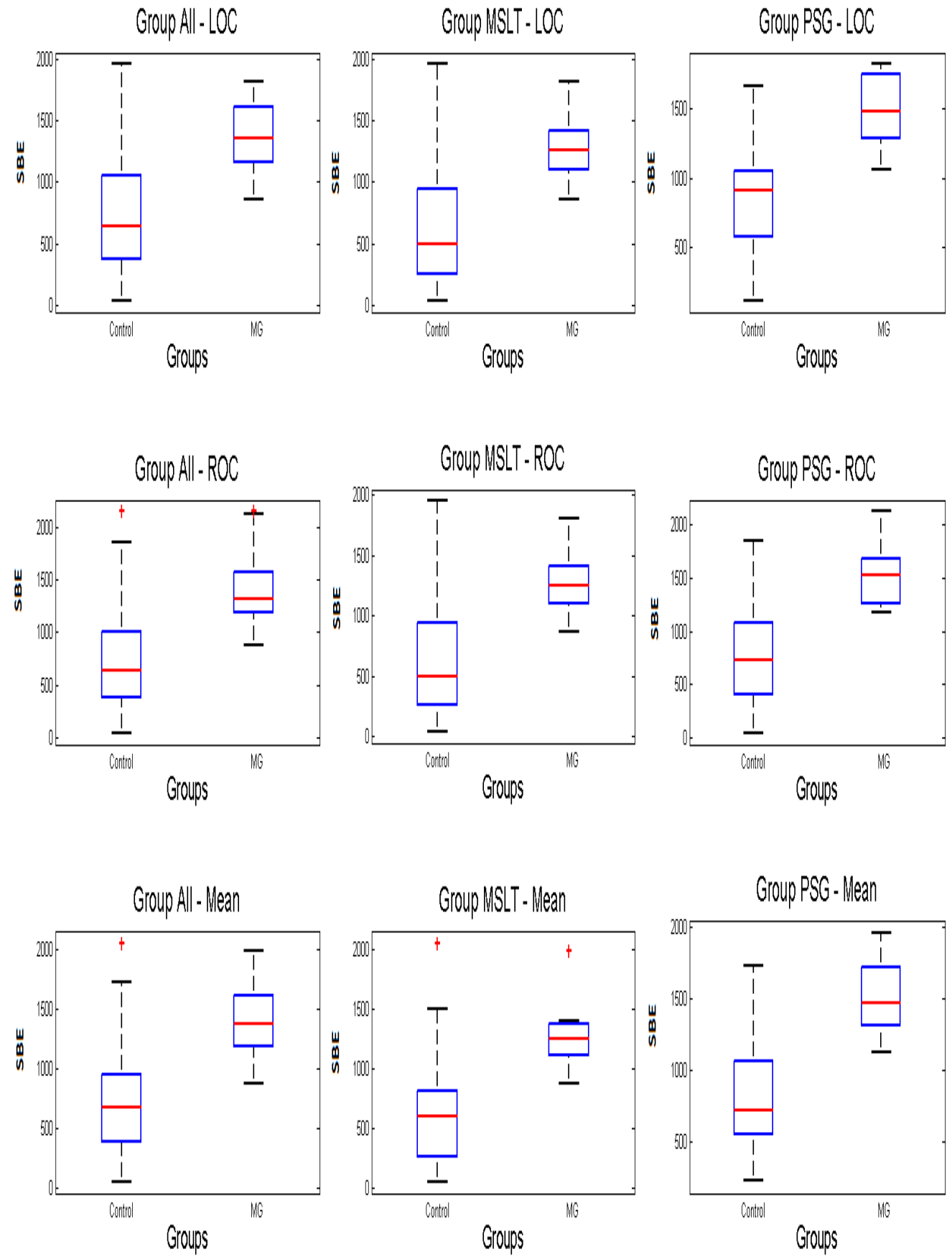

Figure 4.3: Boxplot results for each group using the SBE feature from LOC, ROC, and Mean of the two channels. 
Table 4.1: LOO cross validated classification accuracy using Group All with SBE mean feature (Mexican Hat Wavelet).

\begin{tabular}{|c|c|c|c|c|}
\hline Method & Groups & MG & NC & Total \\
\hline \hline Cross-Validated & MG & $\mathbf{1 4}$ & 2 & 16 \\
\hline & NC & 12 & $\mathbf{5 0}$ & 62 \\
\hline$\%$ & MG & $\mathbf{8 7 . 5}$ & 12.5 & 100 \\
\hline & NC & 19.4 & $\mathbf{8 0 . 6}$ & 100 \\
\hline
\end{tabular}

a stepwise feature selection process, it resulted in choosing the mean SBE to be the best feature. The $\mathrm{F}$ value and $\mathrm{P}$ value were identified to be 32.679 and $2.024 \times 10^{-7}$, respectively. In Table 4.1, shows the classification performance of the mean SBE feature. An overall classification using the LOO cross validation method was $82.1 \%$ with a sensitivity of $87.5 \%$ and specificity of $80.6 \%$ $(\mathrm{P}<<0.01$ i.e. statistically significant). As the overall classification is computed as a weighted sum, which is biased more towards the normal control group, the true accuracy indeed would have been higher than what it appears. An ROC curve was generated based on the classifier to observe its robustness and eliminate the effects of imbalanced data sets. For this particular classifier, the corresponding ROC curve with sensitivity in the $\mathrm{Y}$ axis and specificity in the $\mathrm{X}$ axis was depicted in Figure 4.4. The resultant AUC for this ROC curve was found to be 0.89 .

\subsubsection{Group MSLT}

Following the same procedure as the previous section, a stepwise feature selection process was performed. The analysis displayed that the mean SBE feature was the best suited feature with an $\mathrm{F}$ and $\mathrm{P}$ value of 14.692 and 0.00475 . The LOO based classification accuracies were computed for the MSLT only group (31 control and 8 MG patients) and were tabulated in Table 4.2. We obtained an overall classification accuracy of $87.2 \%$ with a sensitivity of $87.5 \%$ and a specificity of $87.1 \%$ ( $\mathrm{P}<<0.01$ i.e statistically significant). As the sensitivity and specificity are similar in value, the

effects of the imbalanced data set was not as apparent in comparison. In Figure 4.5, it shows the ROC curve with sensitivity in $\mathrm{Y}$ axis and specificity in the $\mathrm{X}$ axis plotted. The AUC is also printed on the figure which is 0.89 . 
Table 4.2: LOO cross validated classification accuracy using Group MSLT with SBE mean feature (Mexican Hat Wavelet).

\begin{tabular}{|c|c|c|c|c|}
\hline Method & Groups & MG & NC & Total \\
\hline \hline Cross-Validated & MG & $\mathbf{7}$ & 1 & 8 \\
\hline & NC & 4 & $\mathbf{2 7}$ & 31 \\
\hline$\%$ & MG & $\mathbf{8 7 . 7}$ & 12.3 & 100 \\
\hline & NC & 12.9 & $\mathbf{8 7 . 1}$ & 100 \\
\hline
\end{tabular}

Table 4.3: LOO cross validated classification accuracy using Group PSG with mean SBE feature (Mexican Hat Wavelet).

\begin{tabular}{|c|c|c|c|c|}
\hline Method & Groups & MG & NC & Total \\
\hline \hline Cross-Validated & MG & $\mathbf{7}$ & 1 & 8 \\
\hline & NC & 6 & $\mathbf{2 5}$ & 31 \\
\hline$\%$ & MG & $\mathbf{8 7 . 5}$ & 12.5 & 100 \\
\hline & NC & 19.4 & $\mathbf{8 0 . 6}$ & 100 \\
\hline
\end{tabular}

\subsubsection{Group PSG}

Using the last data set, the stepwise feature selection process has also identified the mean SBE feature to be the best with an F and P value of 19.780 and 0.000077 . Using the mean SBE as the selected feature, the LOO based classification accuracies were computed for the PSG only group (31 control and $8 \mathrm{MG}$ patients) and was tabulated in Table 4.3. The overall classification accuracy was found to be $82.1 \%$ with a sensitivity of $87.5 \%$ and a specificity of $80.6 \%(\mathrm{P}<<0.01$ i.e statistically significant). Figure 4.6 shows the ROC curve with sensitivity in Y axis and specificity in the $\mathrm{X}$ axis. The AUC for the given classifier was found to be 0.90. The ROC curve and AUC demonstrated high levels of robustness, which testifies to the classifier's overall performance.

Using the classification accuracy with LOO cross validation, the mean SBE feature obtained using the Morlet and Gaussian second order wavelet functions showed similar accuracies. The overall accuracies for Morlet and Gaussian (second order) wavelet functions ranged from 74.4\% to $79.5 \%$ and $79.5 \%$ to $83.3 \%$, respectively. The classification accuracies using the Morlet wavelet for Group All, Group MSLT, and Group PSG are tabulated in Table 4.4, 4.5, and 4.6, respectively. 


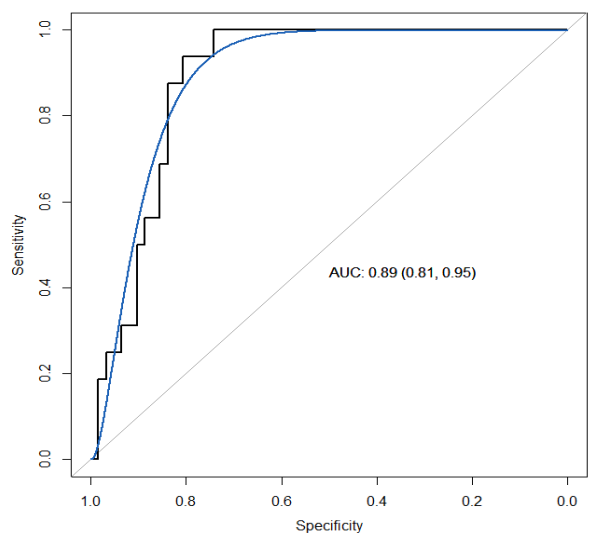

Figure 4.4: ROC curve for classifier using mean SBE for Group All.

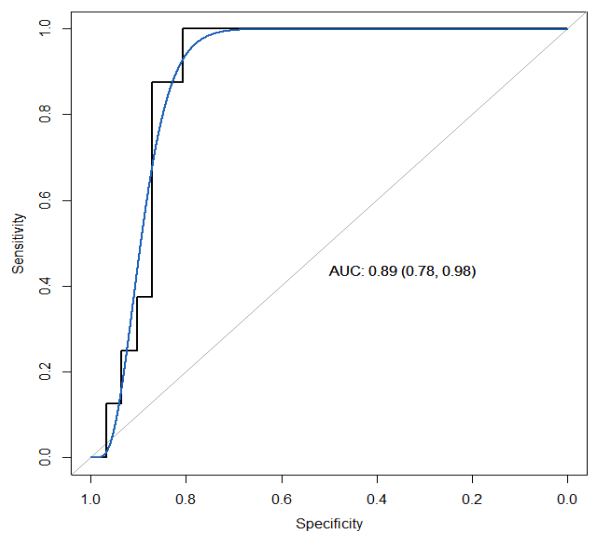

Figure 4.5: ROC curve for classifier using mean SBE for Group MSLT.

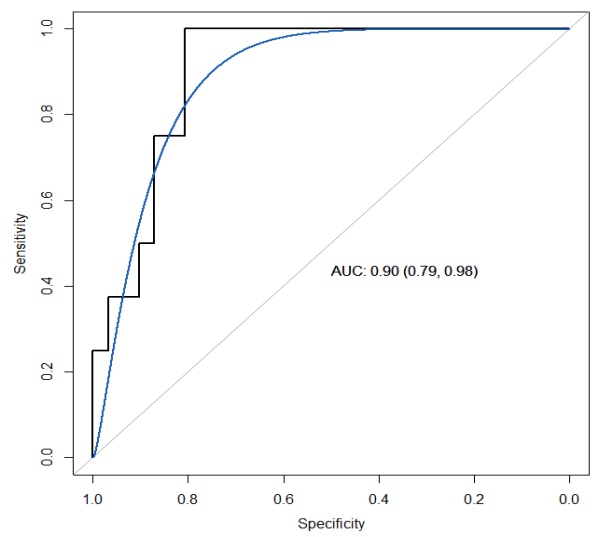

Figure 4.6: ROC curve for classifier using mean SBE for Group PSG. 
Similarly for the Gaussian (second order) wavelet, the classification accuracies for Group All, Group MSLT, and Group PSG are tabulated in Table 4.7, 4.8, and 4.9, respectively. In comparison to the Mexican Hat wavelet function, the classification accuracies obtained by the Morlet and Gaussian (second order) wavelets for each of the 3 data sets were less balanced. The combination of features were also tested and none of the combination produced better results than mean SBE alone.

According to the above presented results, a single time-scale domain feature (mean SBE) could successfully classify MG from control data with or without labels. Observing the boxplots of the distribution of the mean SBE feature, the MG shows a higher mean SBE value than the controls. As this scale band limits the frequency range approximately from 0.8 to $1.42 \mathrm{~Hz}$, the faster SEM were emphasized more. The morphological trait of $\mathrm{MG}$ was shown to frequently have more sharp movements. This translates to the movement being located in higher frequencies or smaller scales more often. Thus, the SBE distribution captured in this particular scale band shows MG being more dominant than the controls.

For the wavelet based features performed well in the classification of MG, the specificity of the technique was lower in comparison to its sensitivity counterpart. As expected from the time domain findings, the scale band energy for MG demonstrated to be greater due to the higher number of sharper movements. With the adaptive wavelet approach, it has eliminated the need for selective threshold parameters to be applied. According to the results, the wavelet approach overall performance was better than the time domain for the given database.

\subsection{Chapter Summary}

In this chapter, the wavelet approach was explored using the same database as the time domain approach. Details regarding the different data sets, pre-processing, feature extraction process, and classification results are presented. Using the stepwise feature selection process, the mean SBE feature was chosen for the analysis. The extracted feature used for the classification demonstrated that the MG group was well separated from the control group. From all 3 data sets, the overall classification accuracies were above $80 \%$ and showed that performance was not dependent on 
Table 4.4: LOO cross validated classification accuracy using Group All with mean SBE feature (Morlet Wavelet).

\begin{tabular}{|c|c|c|c|c|}
\hline Method & Groups & MG & NC & Total \\
\hline \hline Cross-Validated & MG & $\mathbf{1 2}$ & 4 & 16 \\
\hline & NC & 13 & $\mathbf{4 9}$ & 62 \\
\hline$\%$ & MG & $\mathbf{7 5}$ & 25 & 100 \\
\hline & NC & 21 & $\mathbf{7 9}$ & 100 \\
\hline
\end{tabular}

Table 4.5: LOO cross validated classification accuracy using Group MSLT with mean SBE feature (Morlet Wavelet).

\begin{tabular}{|c|c|c|c|c|}
\hline Method & Groups & MG & NC & Total \\
\hline \hline Cross-Validated & MG & $\mathbf{5}$ & 3 & 8 \\
\hline & NC & 5 & $\mathbf{2 6}$ & 31 \\
\hline$\%$ & MG & $\mathbf{6 2 . 5}$ & 37.5 & 100 \\
\hline & NC & 16.1 & $\mathbf{8 3 . 9}$ & 100 \\
\hline
\end{tabular}

Table 4.6: LOO cross validated classification accuracy using Group PSG with mean SBE feature (Morlet Wavelet).

\begin{tabular}{|c|c|c|c|c|}
\hline Method & Groups & MG & NC & Total \\
\hline \hline Cross-Validated & MG & $\mathbf{5}$ & 3 & 8 \\
\hline & NC & 7 & $\mathbf{2 4}$ & 31 \\
\hline$\%$ & MG & $\mathbf{6 2 . 5}$ & 37.5 & 100 \\
\hline & NC & 22.6 & $\mathbf{7 7 . 4}$ & 100 \\
\hline
\end{tabular}


Table 4.7: LOO cross validated classification accuracy using Group All with mean SBE feature (Gaussian 2 Wavelet).

\begin{tabular}{|c|c|c|c|c|}
\hline Method & Groups & MG & NC & Total \\
\hline \hline Cross-Validated & MG & $\mathbf{1 4}$ & 2 & 16 \\
\hline & NC & 11 & $\mathbf{5 1}$ & 62 \\
\hline$\%$ & MG & $\mathbf{8 7 . 5}$ & 12.5 & 100 \\
\hline & NC & 17.7 & $\mathbf{8 2 . 3}$ & 100 \\
\hline
\end{tabular}

Table 4.8: LOO cross validated classification accuracy using Group MSLT with mean SBE feature (Gaussian 2 Wavelet).

\begin{tabular}{|c|c|c|c|c|}
\hline Method & Groups & MG & NC & Total \\
\hline \hline Cross-Validated & MG & $\mathbf{6}$ & 2 & 8 \\
\hline & NC & 5 & $\mathbf{2 6}$ & 31 \\
\hline$\%$ & MG & $\mathbf{7 5}$ & 25 & 100 \\
\hline & NC & 16.1 & $\mathbf{8 3 . 9}$ & 100 \\
\hline
\end{tabular}

Table 4.9: LOO cross validated classification accuracy using Group PSG with mean SBE feature (Gaussian 2 Wavelet).

\begin{tabular}{|c|c|c|c|c|}
\hline Method & Groups & MG & NC & Total \\
\hline \hline Cross-Validated & MG & $\mathbf{6}$ & 2 & 8 \\
\hline & NC & 6 & $\mathbf{2 5}$ & 31 \\
\hline$\%$ & MG & $\mathbf{7 5}$ & 25 & 100 \\
\hline & NC & 19.4 & $\mathbf{8 0 . 6}$ & 100 \\
\hline
\end{tabular}


pre-condition labels. In the wavelet approach, the sensitivity of the technique was higher than specificity. Classification performance was validated with the LOO method due to the small sample size of our database. Additional validation from the ROC curves were performed to eliminate the bias caused by the imbalanced data set. Using the time domain approach results, the rise rate feature led to the finding of the localized scale band, which contained a significant trait unique to MG. The mean SBE prove to be highly effective in classifying MG and control. Considering the signal structure of MG, the Mexican Hat wavelet correlated the best among the explored wavelet functions. 


\section{Chapter 5}

\section{Conclusions and Future Works}

$\mathrm{M}$

YASTHENIA gravis is a neuromuscular disorder that is able to affect anyone and potentially become fatal depending on which group of muscles are involved. Irrespective of the severity of MG, the quality of life of the affected individual will be compromised. The incidence and prevalence rate for MG varies across different regions of the globe and may affect demographics differently. In Ontario from 1996 to 2013, the crude prevalence almost doubled during that time period. As these statistics suggests, MG is progressively becoming an alarming issue. Although treatment plans do exist, the effectiveness of the treatment is dependent on the severity of MG. By diagnosing the MG in its early or initial stages, the progression of the disorder could be prevented. To this effect, this thesis explored the possibility of using electrooculogram (EOG) signals to distinguish MG from control data as initial MG symptoms in most cases are localized in the ocular region. The motivation of this work was to study the traits of MG in hopes of achieving early stage detection. We presented a time domain and a wavelet domain approach in extracting quantifiable features that can classify MG from control data. The time domain approach relied more on manual thresholding methods, which were arrived iteratively to analyze eye movements. In the wavelet approach, we presented an adaptive method in classifying the two groups by tracking the energy captured by specific EOG morphologies localized in a particular scale band. Both approaches were successful in extracting features that were able to separate the MG group with high certainty. 


\subsection{Summary of Results}

In this thesis, three contributions were made. First, the time domain approach in this study revealed that the rise rate of EOG eye movement pulses from SEM was effective in the classification of MG and control groups. For each of the 3 separate data set, the rise rate showed the best classification accuracy. By applying an LDA classifier using the rise rate feature and validating the results with the LOO, 3 separate sets of classification performance were recorded. For the Group All data set, it achieved an overall classification of $83.3 \%$ with a sensitivity of $68.75 \%$ and specificity of $87.1 \%$. In the Group MSLT data set, it achieved an overall classification of $82.1 \%$ with a sensitivity of $62.5 \%$ and specificity of $87.1 \%$. The last data set (Group PSG) achieved an overall classification accuracy of $82.1 \%$ with a sensitivity of $62.5 \%$ and a specificity of $87.1 \%$. Although the sensitivity was low for all the data sets, each individual data set using the rise rate feature displayed a high specificity value. This is significant as it could prove to be useful for clinicians in the screening process of MG. Currently, the RNS diagnostic is used for the screening process, which is semiinvasive. However, our results strongly suggests that the time domain approach using EOG signals could become a viable non-invasive alternative screening method. In comparison to RNS, EOG acquisition is non-invasive and non-intrusive, which is desirable when considering patient care.

The second contribution of this work was from the wavelet based approach in separating the MG and control group. Through scale dependent energy subbanding in the time-scale domain, it was found that the morphology of MG that is able to differentiate it from the control group was located in the frequency range of approximately 0.8 to $1.42 \mathrm{~Hz}$. This was a significant finding because it demonstrated that the unique morphology of MG can be localized in a narrow fre-

quency or scale range. By focusing only on this band, it reduces the complexity of the analysis and shows high discriminating capabilities for the 3 data sets. As different wavelets emphasizes different properties or characteristics of the signal, three different wavelets were selected to the wavelet analysis. Exploring three different wavelet functions, it was found that the Mexican Hat had the best overall classification performance. For the purpose of diagnosing MG, the Mexican Hat wavelet more consistent sensitivity results for the 3 data sets. An LDA classifier was used with LOO as the cross validation method. For the Group All data set, it achieved an overall clas- 
sification of $82.1 \%$ with a sensitivity of $87.5 \%$ and specificity of $80.6 \%$. In the Group MSLT data set, it achieved an overall classification of $87.2 \%$ with a sensitivity of $87.5 \%$ and specificity of 87.1\%. Lastly, the Group PSG data set achieved an overall classification accuracy of $82.1 \%$ with a sensitivity of $87.5 \%$ and a specificity of $80.6 \%$. The significance of these classification results, in contrast to the time domain, was that the sensitivity was higher than the specificity in all cases. This is useful for applications where MG needs to be confirmed in the patient, especially for early stage detection. Through literature, it was understood that MG in the early stages commonly show symptoms of muscular weakness and is localized in the ocular region. With the EOG signals using the wavelet based features for classification, it could help clinicians by making earlier diagnosis of MG. We also tested the combination of the best time domain and wavelet domain features and found them performing with comparable results however, the individual wavelet feature performed overall the best.

Lastly, the results from time domain and wavelet approach both demonstrated that the precondition labels of MSLT and PSG are not required. The significance of this finding was that regardless of which sleep test the patient participates in, the MG morphology can be identified. With early stage MG detection as the end objective, the dependence to use a particular sleep test is eliminated. The other uniqueness of the study is that the entire duration of the test is not required, as the presented results of the analysis were only using a fraction of the sleep tests. Hence, these tests could be shortened to better suit the intended application of screening or diagnosis of MG.

\subsection{Potential Applications}

The following are a few applications of the findings in this thesis:

- Development of non-invasive screening devices for OMG.

- The proposed methods could be valuable in EOG based analysis of other ocular or sleep pathologies, such as nystagmus.

- The method can be extended to perform subclassification of MG based on its severity. 


\subsection{Directions for Future Works}

The end goal of this work was to be able to demonstrate the use of EOG signals as a viable non-invasive alternative for screening MG and early detection. However, as MG can manifest in different forms and has different levels of severity, potential future works could focus on the subclassification of MG. The wavelet analysis results presented in this thesis indicates a huge potential in EOG morphology based characterization of MG, which a new direction to pursue in the analysis of MG. The other direction would be in extending the analysis to sleep studies where clues lie in the ocular region or eye movements. The author hopes that the findings achieved in this work will be applied to further MG studies and contribution will be made in realizing the ability to perform early detection of MG. 


\section{Bibliography}

[1] S. Berrih-Aknin, M. Frenkian-Cuvelier, and B. Eymard. Diagnostic and clinical classification of autoimmune myasthenia gravis. Journal of autoimmunity, 48:143-148, 2014.

[2] J.A. Koch and L.M. Koch. Myasthenia gravis. Journal of gerontological nursing, 39(12):11$5,122013$.

[3] M. de Perrot and L. Donahoe. Thymectomy for myasthenia gravis: whats next? Journal of Thoracic Disease, 9(2):237-239, 2017.

[4] J.P. Bouwyn, P. Magnier, A.L. Bédat-Millet, P. Ahtoy, D. Maltête, and R. Lefaucheur. Antimusk myasthenia gravis with prolonged remission. Neuromuscular Disorders, 26(7):453454, 2016.

[5] S.J. Oh. Muscle-specific receptor tyrosine kinase antibody positive myasthenia gravis current status. Journal of Clinical Neurology, 5(2):53-64, 2009.

[6] N.E. Gilhus, J.F. Owe, J.M. Hoff, F. Romi, G.O. Skeie, and J.A. Aarli. Myasthenia gravis: a review of available treatment approaches. Autoimmune diseases, 2011:1-6, 2011.

[7] S.H. Wong, S. Huda, A. Vincent, and G.T. Plant. Ocular myasthenia gravis: controversies and updates. Current neurology and neuroscience reports, 14(1):421, 2014.

[8] M.J. Kupersmith. Ocular myasthenia gravis: treatment successes and failures in patients with long-term follow-up. Journal of neurology, 256(8):1314-1320, 2009.

[9] A.G. Nair, P. Patil-Chhablani, D.V. Venkatramani, and R.A. Gandhi. Ocular myasthenia gravis: A review. Indian journal of ophthalmology, 62(10):985, 2014. 
[10] N.E. Gilhus. Autoimmune myasthenia gravis. Expert review of neurotherapeutics, 9(3):351$358,2009$.

[11] M.N. Meriggioli and D.B. Sanders. Autoimmune myasthenia gravis: emerging clinical and biological heterogeneity. The Lancet Neurology, 8(5):475-490, 2009.

[12] E. Santos, E. Coutinho, I. Moreira, A.M. Silva, D. Lopes, H. Costa, F. Silveira, G. Nadais, H. Morais, J. Martins, et al. Epidemiology of myasthenia gravis in northern portugal: Frequency estimates and clinical epidemiological distribution of cases. Muscle \& nerve, 54(3):413-421, 2016.

[13] A. Breiner, J. Widdifield, H.D. Katzberg, C. Barnett, V. Bril, and K. Tu. Epidemiology of myasthenia gravis in ontario, canada. Neuromuscular Disorders, 26(1):41-46, 2016.

[14] N.E. Gilhus, A. Nacu, J.B. Andersen, and J.F. Owe. Myasthenia gravis and risks for comorbidity. European journal of neurology, 22(1):17-23, 2015.

[15] D. Grob, N. Brunner, T. Namba, and M. Pagala. Lifetime course of myasthenia gravis. Muscle \& nerve, 37(2):141-149, 2008.

[16] J. Gamez and J.M. Ponseti. Thymectomy for non-thymomatous myasthenia gravis: the end of controversy, albeit fifty years late. Journal of Xiangya Medicine, 2(1), 2017.

[17] A. Masaoka, Y. Yamakawa, H. Niwa, I. Fukai, S. Kondo, M. Kobayashi, Y. Fujii, and Y. Monden. Extended thymectomy for myasthenia gravis patients: a 20-year review. The Annals of thoracic surgery, 62(3):853-859, 1996.

[18] N.M. Alkhawajah and J. Oger. Treatment of myasthenia gravis in the aged. Drugs \& aging, 32(9):689-697, 2015.

[19] E. Kerty, A. Elsais, Z. Argov, A. Evoli, and N.E. Gilhus. Efns/ens guidelines for the treatment of ocular myasthenia. European journal of neurology, 21(5):687-693, 2014. 
[20] L.A. Jones and N.P. Robertson. An update on treatments in myasthenia gravis. Journal of Neurology, 264(1):205-207, 2017.

[21] L. Padua, P. Caliandro, G. Di Iasi, C. Pazzaglia, F. Ciaraffa, and A. Evoli. Reliability of sfemg in diagnosing myasthenia gravis: sensitivity and specificity calculated on 100 prospective cases. Clinical Neurophysiology, 125(6):1270-1273, 2014.

[22] J.J. Plomp, M. Morsch, W.D. Phillips, and J.J.G.M. Verschuuren. Electrophysiological analysis of neuromuscular synaptic function in myasthenia gravis patients and animal models. Experimental neurology, 270:41-54, 2015.

[23] M. Liik and A.R. Punga. Repetitive nerve stimulation often fails to detect abnormal decrement in acute severe generalized myasthenia gravis. Clinical Neurophysiology, 127(11):3480-3484, 2016.

[24] A. Abraham, M. Alabdali, A. Alsulaiman, A. Breiner, C. Barnett, H.D. Katzberg, L.E. Lovblom, and V. Bril. Repetitive nerve stimulation cutoff values for the diagnosis of myasthenia gravis. Muscle \& nerve, 55(2):166-170, 2016.

[25] H.B. Ali, E. Salort-Campana, A.M. Grapperon, J. Gallard, J. Franques, A. Sevy, E. Delmont, A. Verschueren, J. Pouget, and S. Attarian. New strategy for improving the diagnostic sensitivity of repetitive nerve stimulation in myasthenia gravis. Muscle \& Nerve, 2017.

[26] J.J.S. Barton. Quantitative ocular tests for myasthenia gravis: a comparative review with detection theory analysis. Journal of the neurological sciences, 155(1):104-114, 1998.

[27] Y. Valko, S.M. Rosengren, H.H. Jung, D. Straumann, K. Landau, and K.P. Weber. Ocular vestibular evoked myogenic potentials as a test for myasthenia gravis. Neurology, 86(7):660668, 2016.

[28] R.B. Reilly and T.C. Lee. Electrograms (ecg, eeg, emg, eog). Technology and Health Care, 18(6):443-458, 2010. 
[29] R. Barea, L. Boquete, S. Ortega, E. López, and J.M. Rodríguez-Ascariz. Eog-based eye movements codification for human computer interaction. Expert Systems with Applications, 39(3):2677-2683, 2012.

[30] L.Y. Deng, C.L. Hsu, T.C. Lin, J.S. Tuan, and S.M. Chang. Eog-based human-computer interface system development. Expert Systems with Applications, 37(4):3337-3343, 2010.

[31] G. Westheimer. The law of equal innervation of both eyes: Thomas reid preceded hering by a century. an historical note. Vision research, 101:32-33, 2014.

[32] C.A. Kushida, M.R. Littner, T. Morgenthaler, C.A. Alessi, D. Bailey, J. Coleman Jr, L. Friedman, M. Hirshkowitz, S. Kapen, M. Kramer, et al. Practice parameters for the indications for polysomnography and related procedures: an update for 2005. Sleep, 28(4):499-521, 2005.

[33] V.K. Kapur, D.H. Auckley, S. Chowdhuri, D.C. Kuhlmann, R. Mehra, K. Ramar, and C.G. Harrod. Clinical practice guideline for diagnostic testing for adult obstructive sleep apnea: An american academy of sleep medicine clinical practice guideline. J Clin Sleep Med, 13(3):479504, 2017.

[34] Standards of Practice Committee of the American Academy of Sleep Medicine et al. Practice parameters for clinical use of the multiple sleep latency test and the maintenance of wakefulness test. Sleep, 28(1):113-121, 2005.

[35] S.S. Sullivan and C.A. Kushida. Multiple sleep latency test and maintenance of wakefulness test. CHEST Journal, 134(4):854-861, 2008.

[36] R. Barea, L. Boquete, M. Mazo, and E. López. Wheelchair guidance strategies using eog. Journal of Intelligent \& Robotic Systems, 34(3):279-299, 2002.

[37] W.M.B.W Daud and R. Sudirman. Time frequency analysis of electrooculograph (eog) signal of eye movement potentials based on wavelet energy distribution. In Modelling Symposium (AMS), 2011 Fifth Asia, pages 81-86. IEEE, 2011. 
[38] A.B. Usakli, S. Gurkan, F. Aloise, G. Vecchiato, and F. Babiloni. On the use of electrooculogram for efficient human computer interfaces. Computational intelligence and neuroscience, 2010:1-5, 2010.

[39] G. Tedeschi, A. Quattrone, and V. Bonavita. Saccadic eye movements analysis as a measure of drug effect on central nervous system function. The Italian Journal of Neurological Sciences, 7(2):223-231, 1986.

[40] P. Stavrou, P.A. Good, E.J. Broadhurst, S. Bundey, A.R. Fielder, and S.J. Crews. Erg and eog abnormalities in carriers of x-linked retinitis pigmentosa. Eye, 10(5):581-589, 1996.

[41] A.J.L.G. Pinckers, M.H.M. Cuypers, and A.L. Aandekerk. The eog in best's disease and dominant cystoid macular dystrophy (demd). Ophthalmic genetics, 17(3):103-108, 1996.

[42] P. Shokrollahi, S. Krishnan, K. Umapathy, K. McConville, M.I. Boulos, D. Jewell, and B.J. Murray. A method for quantifying sleep eye movements that reflects medication effects. In World Congress on Medical Physics and Biomedical Engineering, September 7-12, 2009, Munich, Germany, pages 1411-1414. Springer, 2010.

[43] J. Virkkala, J. Hasan, A. Värri, S.L. Himanen, and K. Müller. Automatic sleep stage classification using two-channel electro-oculography. Journal of neuroscience methods, 166(1):109$115,2007$.

[44] M.E. Tagluk, N. Sezgin, and M. Akin. Estimation of sleep stages by an artificial neural network employing eeg, emg and eog. Journal of medical systems, 34(4):717-725, 2010.

[45] B.J. Swihart, B. Caffo, K. Bandeen-Roche, and N.M. Punjabi. Characterizing sleep structure using the hypnogram. J Clin Sleep Med, 4(4):349-355, 2008.

[46] D. Shin, H. Sakai, and Y. Uchiyama. Slow eye movement detection can prevent sleep-related accidents effectively in a simulated driving task. Journal of sleep research, 20(3):416-424, 2011. 
[47] M.I. Boulos, K. Umapathy, P. Shokrollahi, K.M.V. McConville, T. Sudenis, D.R. Jewell, S. Krishnan, and B.J. Murray. Automated detection of nocturnal slow eye movements modulated by selective serotonin reuptake inhibitors. Progress in Neuro-Psychopharmacology and Biological Psychiatry, 35(1):126-130, 2011.

[48] A. Bulling, J.A. Ward, H. Gellersen, and G. Troster. Eye movement analysis for activity recognition using electrooculography. IEEE transactions on pattern analysis and machine intelligence, 33(4):741-753, 2011.

[49] M. Nakanishi, Y. Mitsukura, Y. Wang, Y.T. Wang, and T.P. Jung. Online voluntary eye blink detection using electrooculogram. In International Symposium on Nonlinear Theory and Its Applications, Palma, Mallorca, Spain, 2012.

[50] R.X. Gao and R. Yan. Wavelets: Theory and applications for manufacturing. Springer Science \& Business Media, 2010.

[51] J. Rafiee, M.A. Rafiee, N. Prause, and M.P. Schoen. Wavelet basis functions in biomedical signal processing. Expert Systems with Applications, 38(5):6190-6201, 2011.

[52] L. Angrisani, P. Daponte, M. D’apuzzo, and A. Testa. A measurement method based on the wavelet transform for power quality analysis. IEEE Transactions on Power Delivery, 13(4):990-998, 1998.

[53] P.J. Winklewski, M. Gruszecki, J. Wolf, E. Swierblewska, K. Kunicka, M. WszedybylWinklewska, W. Guminski, J. Zabulewicz, A.F. Frydrychowski, L. Bieniaszewski, et al. Wavelet transform analysis to assess oscillations in pial artery pulsation at the human cardiac frequency. Microvascular research, 99:86-91, 2015.

[54] S.P. Singh and S. Urooj. Wavelets: biomedical applications. International Journal of Biomedical Engineering and Technology, 19(1):1-25, 2015.

[55] S. Mallat. A wavelet tour of signal processing: the sparse way. Academic press, 2008. 
[56] L. Marple. Computing the discrete-time" analytic" signal via fft. IEEE Transactions on signal processing, 47(9):2600-2603, 1999.

[57] O.A. Rosso, S. Blanco, J. Yordanova, V. Kolev, A. Figliola, M. Schürmann, and E. Başar. Wavelet entropy: a new tool for analysis of short duration brain electrical signals. Journal of neuroscience methods, 105(1):65-75, 2001.

[58] W.J. Staszewski. Identification of non-linear systems using multi-scale ridges and skeletons of the wavelet transform. Journal of Sound and Vibration, 214(4):639-658, 1998.

[59] J.M. Lilly and J.C. Gascard. Wavelet ridge diagnosis of time-varying elliptical signals with application to an oceanic eddy. Nonlinear Processes in Geophysics, 13(5):467, 2006.

[60] S. Li, X. Su, and W. Chen. Wavelet ridge techniques in optical fringe pattern analysis. JOSA A, 27(6):1245-1254, 2010.

[61] M. Unser and A. Aldroubi. A review of wavelets in biomedical applications. Proceedings of the IEEE, 84(4):626-638, 1996.

[62] R.M. Rangayyan. Biomedical signal analysis, volume 33. John Wiley \& Sons, 2015.

[63] M.S. Reddy, B. Narasimha, E. Suresh, and K.S. Rao. Analysis of eog signals using wavelet transform for detecting eye blinks. In Wireless Communications and Signal Processing (WCSP), 2010 International Conference on, pages 1-4. IEEE, 2010.

[64] E. Magosso, M. Ursino, F. Provini, and P. Montagna. Wavelet analysis of electroencephalographic and electro-oculographic changes during the sleep onset period. In Engineering in Medicine and Biology Society, 2007. EMBS 2007. 29th Annual International Conference of the IEEE, pages 4006-4010. IEEE, 2007.

[65] R.O. Duda, P.E. Hart, and D.G. Stork. Pattern classification. John Wiley \& Sons, 2012.

[66] C.E. Metz. Basic principles of roc analysis. In Seminars in nuclear medicine, volume 8, pages 283-298. Elsevier, 1978. 
[67] T. Fawcett. An introduction to roc analysis. Pattern recognition letters, 27(8):861-874, 2006.

[68] T. Liang, M.I. Boulos, B.J. Murray, S. Krishnan, H. Katzberg, and K. Umapathy. Detection of myasthenia gravis using electrooculography signals. In Engineering in Medicine and Biology Society (EMBC), 2016 IEEE 38th Annual International Conference, pages 896-899. IEEE, 2016.

[69] The Mathworks, Inc., Natick, Massachusetts. version 8.1.0.604 (R2013a), 2013.

[70] IBM Corp., Armonk, NY:IBM Corp. IBM SPSS Statistics for Windows Version 24.0, 2016.

[71] J.R. Deatherage, R.D. Roden, and K. Zouhary. Normal sleep architecture. In Seminars in orthodontics, volume 15, pages 86-87. Elsevier, 2009.

[72] A. Banerjee, M. Pal, D.N. Tibarewala, and A. Konar. Electrooculogram based blink detection to limit the risk of eye dystonia. In Advances in Pattern Recognition (ICAPR), 2015 Eighth International Conference on, pages 1-6. IEEE, 2015.

[73] M. Fabbri, F. Pizza, E. Magosso, M. Ursino, S. Contardi, F. Cirignotta, F. Provini, and P. Montagna. Automatic slow eye movement (sem) detection of sleep onset in patients with obstructive sleep apnea syndrome (osas): comparison between multiple sleep latency test (mslt) and maintenance of wakefulness test (mwt). Sleep medicine, 11(3):253-257, 2010.

[74] C.A. Joyce, I.F. Gorodnitsky, and M. Kutas. Automatic removal of eye movement and blink artifacts from eeg data using blind component separation. Psychophysiology, 41(2):313-325, 2004.

[75] R. Agarwal, T. Takeuchi, S. Laroche, and J. Gotman. Detection of rapid-eye movements in sleep studies. IEEE Transactions on biomedical engineering, 52(8):1390-1396, 2005.

[76] M. Rasooli, F.H. Foomany, K. Balasundaram, S. Masse, N. Zamiri, A. Ramadeen, X. Hu, P. Dorian, K. Nanthakumar, S. Krishnan, et al. Analysis of electrocardiogram pre-shock waveforms during ventricular fibrillation. Biomedical Signal Processing and Control, 21:2633, 2015. 